\title{
Scattering Properties of Large Irregular Cosmic Dust Particles at Visible Wavelengths
}

\section{Escobar-Cerezo, J.}

2017-03-20

Escobar-Cerezo , J , Palmer , C , Munoz , O , Moreno , F , Penttilä , A \& Muinonen , K 2017 , ' Scattering Properties of Large Irregular Cosmic Dust Particles at Visible Wavelengths ', Astrophysical Journal , vol. 838 , no. 1 , 74 . https://doi.org/10.3847/1538-4357/aa6303

unspecified

publishedVersion

Downloaded from Helda, University of Helsinki institutional repository.

This is an electronic reprint of the original article.

This reprint may differ from the original in pagination and typographic detail.

Please cite the original version. 


\title{
Scattering Properties of Large Irregular Cosmic Dust Particles at Visible Wavelengths
}

\author{
J. Escobar-Cerezo ${ }^{1}$, C. Palmer ${ }^{1}$, O. Muñoz ${ }^{1}$, F. Moreno ${ }^{1}$, A. Penttilä ${ }^{2}$, and K. Muinonen ${ }^{2,3}$ \\ ${ }^{1}$ Instituto de Astrofissica de Andalucìa, CSIC, Glorieta de la Astronomìa s/n, E-18008 Granada, Spain \\ ${ }^{2}$ Department of Physics, P.O. Box 64, FI-00014 University of Helsinki, Finland \\ ${ }^{3}$ National Land Survey of Finland, Finnish Geospatial Research Institute, P.O. Box 84, FI-00521 Helsinki, Finland \\ Received 2016 October 14; revised 2017 February 21; accepted 2017 February 22; published 2017 March 27
}

\begin{abstract}
The effect of internal inhomogeneities and surface roughness on the scattering behavior of large cosmic dust particles is studied by comparing model simulations with laboratory measurements. The present work shows the results of an attempt to model a dust sample measured in the laboratory with simulations performed by a ray-optics model code. We consider this dust sample as a good analogue for interplanetary and interstellar dust as it shares its refractive index with known materials in these media. Several sensitivity tests have been performed for both structural cases (internal inclusions and surface roughness). Three different samples have been selected to mimic inclusion/coating inhomogeneities: two measured scattering matrices of hematite and white clay, and a simulated matrix for water ice. These three matrices are selected to cover a wide range of imaginary refractive indices. The selection of these materials also seeks to study astrophysical environments of interest such as Mars, where hematite and clays have been detected, and comets. Based on the results of the sensitivity tests shown in this work, we perform calculations for a size distribution of a silicate-type host particle model with inclusions and surface roughness to reproduce the experimental measurements of a dust sample. The model fits the measurements quite well, proving that surface roughness and internal structure play a role in the scattering pattern of irregular cosmic dust particles.
\end{abstract}

Key words: comets: general - methods: numerical - planets and satellites: atmospheres - polarization - scattering

\section{Introduction}

Dust can be found in many different environments in the universe, from being a component of the interstellar medium to forming the regolith and atmospheres of solar system bodies. As an example of its distant detection and study, optical images of the nearby star Fomalhaut show a ring of dust orbiting the central star. This dust is expected to be similar in several properties to the zodiacal dust in our solar system (Min et al. 2010), so through its study we can better understand our vicinity. Moreover, the scattered light by this system could be dominated by large dust grains of at least $100 \mu \mathrm{m}$. Dust grains are the building blocks of planetary systems. In the core accretion disk surrounding young stars, grains typically grow through collisions, from sub-micron-size particles into larger aggregates until these aggregates reach planetesimal sizes. This growth is limited to micron sizes in dense regions of molecular clouds, but it is possible for them to grow further in the midplane of protoplanetary disks (Testi et al. 2014). Here, micronsize dust grains coagulate to form larger structures with complex shapes and compositions. This process significantly changes the optical properties of the scattering particles. To properly interpret observations of protoplanetary disks and to place these observations in the context of the early stages of planet formation, it is crucial to understand the optical properties of these complex structures (Min et al. 2016).

Mineral dust is also present in many solar system bodies. In planetary atmospheres, suspended grains play an important role in the radiative transfer of incident solar energy and act as condensation and freezing nuclei within the water cycle. Dust is also found in comets and the surface of atmosphere-less satellites. Recently, the Rosetta mission has studied in situ, among other things, dust properties of both the nucleus surface and coma of the comet 67P/Churyumov-Gerasimenko, thanks to instruments such as the Grain Impact Analyzer and Dust
Accumulator (GIADA) and the Optical, Spectroscopic, and Infrared Remote Imaging System (OSIRIS). GIADA was able to distinguish different types of particles populating the coma of 67P. These particles can be separated into two families: compact particles (ranging in size from 0.03 to $1 \mathrm{~mm}$ ), which underwent processing within the solar nebula, and fluffy aggregates (ranging in size from 0.2 to $2.5 \mathrm{~mm}$ ) of sub-micron grains, which might be a record of a primitive component, probably linked to interstellar dust (Fulle et al. 2015). Some physical processes related to comets depend strongly on the size distribution of dust particles in their atmospheres, surfaces, and the first few meters below (Fulle et al. 2016). It is also important to know the spatial distribution of grains in the coma as illustrated in Della Corte et al. (2015) for comet 67P/ Churyumov-Gerasimenko. The authors determined the dynamical and physical properties of cometary dust particles to support the study of the production process and dust environment evolution.

In addition to observations and laboratory measurements of scattering patterns of dust particles, several computational codes have been developed in the last couple of decades to simulate their scattering behavior, which depends on a broad range of physical properties of the grains. In early investigations, these particles were assumed to be spheres so as to simplify the calculations. Electromagnetic scattering from spherical particles is described analytically by Mie theory (Mie 1908), which provides an exact solution to the problem. Unfortunately, this approach proved to be unfruitful in many applications as the simulations reproduced neither the observations nor experimental measurements (Mishchenko et al. 2003). Further refinements were made; instead of spheres, cylinders were used in an attempt to introduce more asymmetry in the particles according to the irregularity of natural dust, but despite this refinement, the results were not as good as expected 
(Wolff et al. 2006, 2010). The problem is that dust particles are highly irregularly shaped, with a high variety of surface roughness and internal cavities and inhomogeneities.

Several attempts have been made in the past to find a way to reproduce experimental measurements and observations of large irregular particles by means of computer simulations (Draine \& Flatau 1994; Mishchenko et al. 2000; Kahnert 2003; Min et al. 2005a; Mishchenko 2009; Muinonen et al. 2009; Nousiainen 2009; Nousiainen et al. 2011; Zubko et al. 2013). The ray-optics approximation method (ROA) is applicable to particles much larger than the incident wavelength and tries to mimic scattering by tracing ray trajectories through the dust grain (Muinonen et al. 1996). In our approach, these particles are simulated using Gaussian Random Spheres (GRSs), as described in Section 4.2. ROA computes separately the scattering produced by forward diffraction and geometrical optics. The forward diffraction computation takes into account the two-dimensional silhouette of each sample shape. For the geometric optics part, every ray is related to a Stokes vector and, once the ray reaches the surface, reflection and refraction are evaluated according to Fresnel's equation and Snell's law. Although ROA does a significantly better job at reproducing scattering by dust particles than simpler models, like Mie theory, some fine tuning is required by the user.

It is not only the grain's shape but also the presence of wavelength-scale surface roughness which affects scattering properties (Kemppinen et al. 2015). Indeed, the surface roughness seems to be responsible for the phase reddening (increasing spectral slope with increasing solar phase angle) observed in Mars (Schröder et al. 2014). Because of this, the next step in the refinement of phenomenological scattering models was to simulate surface roughness and internal inhomogeneities using simple schemes of Lambertian surface elements and internal screens (Nousiainen et al. 2003). The comparison of the experimental scattering matrix of a dust sample consisting of particles larger than the wavelength with calculations of a ray-optics method employing GRSs has made it clear that the single-scattering properties of some samples of irregular particles cannot be accurately modeled without accounting for the effects of surface roughness (Muñoz et al. 2006). More sophisticated models have since been made in response (Muinonen et al. 2009). The results were promising, but insufficient to reproduce experimental measurements. Subsequently, ROA evolved into Ray Optics with Diffuse and Specular interactions (RODSs), which is explained in Section 4. RODS includes the effects of more detailed features such as wavelength-scale internal inhomogeneities and surface roughness, characterized by additional scattering matrices. As a first approach, synthetic matrices resembling the scattering of certain materials were used to model wavelength-scale surface roughness, obtaining promising results in the field.

In this work we study for the first time the effect of using experimental scattering matrices to mimic internal and external inhomogeneities in particles larger than the wavelength of the incident light. Moreover, we also perform a parameter space exploration on the particle size and volume of inhomogeneities (both internal inclusions and surface roughness) to study their effect on the computed scattering matrix elements. All computations presented in this work are performed at a wavelength of $632.8 \mathrm{~nm}$. The performance of RODS is tested by direct comparison with the experimental scattering matrix of a dust sample with optical properties (refractive index) similar to enstatite, a free-iron form of pyroxene found in both interplanetary and interstellar media. Detailed information about these samples is presented in Section 3.

This article is structured as follows: in Section 2 we present a brief review of the basic scattering concepts; Section 3 gives a description and a discussion of the physical properties of the samples; the ROA and RODS code are explained in Section 4; sensitivity tests and the results of simulations to fit experimental data are presented in Section 5; and finally, a summary of the results and the conclusions are presented in Section 6.

\section{Basic Concepts}

The polarization state of a light beam can be characterized by the Stokes vector and its parameters, $I, Q, U$, and $V$, known as the Stokes parameters. An incident beam $\pi \boldsymbol{\Phi}_{0}(\lambda)$ is related to the scattered beam $\pi \boldsymbol{\Phi}_{\mathrm{det}}(\lambda, \theta)$ through the $4 \times 4$ scattering matrix, $F$, for a sample of randomly oriented particles, where $\theta$ is the angle between the propagation directions of incident and scattered light. This matrix has the form (Hovenier et al. 2004):

$$
\boldsymbol{\Phi}_{\text {det }}(\lambda, \theta)=\frac{C_{\text {sca }}}{4 \pi^{2} D^{2}}\left(\begin{array}{cccc}
F_{11} & F_{12} & F_{13} & F_{14} \\
F_{12} & F_{22} & F_{23} & F_{24} \\
-F_{13} & -F_{23} & F_{33} & F_{34} \\
F_{14} & F_{24} & -F_{34} & F_{44}
\end{array}\right) \boldsymbol{\Phi}_{0}(\lambda, \theta),
$$

where the first elements of the column vectors are fluxes divided by $\pi$, and the state of polarization of the beams is described by the other Stokes parameters. Furthermore, $\lambda$ is the wavelength, $C_{\text {sca }}$ is the scattering cross section (rate of energy scattered by the sample), and $D$ is the distance from the sample to the detector. The plane containing the directions of the incident and scattered beams, known as the scattering plane, is the plane of reference for the flux vectors. The $F_{i j}$ elements are dimensionless and depend on particle physical properties, as size, shape, and refractive index, and other parameters as the number of the scattering particles that contribute to the detected radiation, the wavelength of the radiation, and the direction of the scattered light, which is sufficiently described by means of the scattering angle $\theta$ for randomly oriented particles. The matrices are normalized such that

$$
\frac{1}{4 \pi} \int_{4 \pi} F_{11} d \Omega=1,
$$

where $\Omega$ is the solid angle. The $F_{11}(\theta)$ normalized in this way is called the phase function. The usual way to represent the scattering matrix elements is by dividing $F_{i j}(\theta)$ elements by $F_{11}(\theta)$, for $i, j=1,2,3,4$ except for $i=j=1$. For $F_{11}(\theta)$ we will use the relative phase function expression $F_{11}(\theta) / F_{11}\left(30^{\circ}\right)$ when dealing with size distributions for comparison with measurements. For those sensitivity tests involving individual sizes (no size distribution integration), we maintain the $F_{11}(\theta)$ normalized as presented in Equation (2). Also, for unpolarized light, the ratio $-F_{12}(\theta) / F_{11}(\theta)$ is called the degree of linear polarization of the scattered light.

The scattering matrix for a given particle depends on the particle size relative to the wavelength of incident light. This is expressed by the size parameter, $x$ :

$$
x=\frac{2 \pi a}{\lambda},
$$


where $a$ is the mean radius of the particle.

The rates of energy scattered and absorbed by single particles are expressed by scattering and absorption cross sections, $C_{\mathrm{sca}}$ and $C_{\text {abs. }}$. These quantities have dimensions of length squared and relate the power scattered and absorbed to a normal surface area upon which equal power is incident. The total attenuation of incident power is the sum of $C_{\mathrm{sca}}$ and $C_{\mathrm{abs}}$ and is called the extinction cross section $C_{\text {ext }}$. The singlescattering albedo can be described as the fraction of light that is scattered over that which is extinguished:

$$
\varpi=\frac{C_{\text {sca }}}{C_{\text {ext }}}=\frac{C_{\text {sca }}}{C_{\text {sca }}+C_{\text {abs }}} .
$$

The angular distribution of scattered power can be conveniently characterized by the so-called asymmetry parameter:

$$
g=\frac{1}{4 \pi} \int_{4 \pi} F_{11} \cos \theta d \Omega,
$$

where $\theta$ is the scattering angle. The asymmetry parameter is often used, e.g., in atmospheric energy balance considerations, due to its connection to the amount of power scattered back to space.

In this work, every simulation consists of a bulk particle, also known as the host particle, which has a certain size and shape as determined by the choice of GRS parameters. The host particle can contain additional scattering elements with different optical properties to the host, so as to simulate the effect of surface roughness and internal inclusions.

To characterize internal inhomogeneities and structure, a diffuse internal medium (DIM) can be added. We use the single-scattering albedo, $\varpi_{\text {DIM }}$, of the DIM material and the mean free-path length of interaction, $l_{o}$, to describe the internal structure. In RODS, $l_{o}$ is related to the volume fraction of diffuse internal scatterers, and is a measure of the mean length that an electromagnetic wave can travel inside the host material between diffuse scattering events. For internal scatterers of radius $r$ and volume fraction $\rho_{\text {int }}$, the mean free path, $l_{o}$, can be calculated by

$$
l_{o}=\frac{4 r_{\mathrm{DIM}}}{3 \rho_{\mathrm{int}} Q_{\mathrm{ext}}},
$$

where $Q_{\text {ext }}$ is the extinction efficiency. In our case we compute $Q_{\text {ext }}$ by applying Mie theory for small homogeneous spheres of radius $r_{\text {DIM }}$ with a refractive index equal to that of the desired inclusion material. Please note that the scatterers are dimensionless in the code: the purpose of $r_{\mathrm{DIM}}$ is just to evaluate $l_{o}$.

The surface roughness, or diffuse external medium (DEM), is defined in this work through the albedo of the DEM, $\varpi_{\text {DEM }}$, and the optical thickness $\tau_{\text {ext }}$, which represents the extinction suffered by electromagnetic waves when transmitted through the host particle surface. The amount of energy that travels through the surface layer unaltered, known as transmittance $T$, can be described by

$$
T=e^{-\tau_{\mathrm{ext}}} .
$$

\section{Sample and Experimental Data}

The main objective of this work is to test the RODS approach for reproducing the effect of internal inhomogeneities and wavelength-scale surface roughness on the optical properties of cosmic dust grains much larger than the wavelength of incident light. To do this, we try to model the experimental scattering matrix elements as functions of the scattering angle of a Saharan desert (Libyan) dust sample (Muñoz et al. 2007), which from now on we refer to as dust sample. The measurements of this sample performed at $\lambda=632.8 \mathrm{~nm}$ are freely available at the Amsterdam-Granada Light Scattering Database (Muñoz et al. 2012) (http://www.iaa.es/scattering/). Moreover, as detailed below, this sample consists of particles larger than $\lambda$, which makes it an ideal test case for the performance of RODS. Based on published refractive indices (Patterson et al. 1977; Dubovik et al. 2006; Shettle \& Fenn 1979), we estimate an average refractive index of our experimental sample, $m_{\text {sample }}=1.5+i 0.0004$, at $\lambda=632.8 \mathrm{~nm}$. This refractive index is similar to that found in free-iron pyroxene, i.e., enstatite $\left(\mathrm{MgSiO}_{3}, m_{\text {enstatite }}=1.569+\right.$ $i 2.6 \times 10^{-5}$ for $\lambda=650 \mathrm{~nm}$ ) (Dorschner et al. 1995). Enstatite has been found in protoplanetary debris disks around young stars (Fujiwara et al. 2010) and evolved stars (Molster et al. 2002), and has been proposed through computational modeling as a component, among other silicates, of the HaleBopp dust coma (Min et al. 2005b). For these reasons we consider this sample as a good analogue for silicate species found in interplanetary and interstellar media.

Size is an important parameter to consider when determining the scattering properties of small particles. To simplify the description of the size distribution, each particle is replaced by a sphere of radius $r$, with a projected surface area equal to the averaged value over all orientations. Some of the results presented later depend on a size distribution of particles. RODS can compute one single size per simulation, so we perform simulations for various particle sizes following the measured size distribution of the sample. The values of this size distribution are presented and studied in Muñoz et al. (2007) and are available in the Amsterdam-Granada Light Scattering Database (Muñoz et al. 2012). From the retrieved number distribution we obtained the values of the effective radius, $r_{\text {eff }}$, and effective variance, $v_{\text {eff }}$, defined as follows (Hansen \& Travis 1974):

$$
\begin{gathered}
r_{\mathrm{eff}}=\frac{\int_{0}^{\infty} r \pi r^{2} n(r) d r}{\int_{0}^{\infty} \pi r^{2} n(r) d r}, \\
v_{\mathrm{eff}}=\frac{\int_{0}^{\infty}\left(r-r_{\mathrm{eff}}\right) \pi r^{2} n(r) d r}{r_{\mathrm{eff}}^{2} \int_{0}^{\infty} \pi r^{2} n(r) d r} .
\end{gathered}
$$

The effective radius and variance for the dust sample are $r_{\text {eff }}=125 \mu \mathrm{m}$ and $v_{\text {eff }}=0.15$, which makes it an ideal test case for the performance of RODS.

Some examples of images of particles in the sample, taken with a Field Emission Scanning Electron Microscope, are presented in Figure 1. The particles have round shapes with occasional sharp edges (Figure 1, left panel). In Figure 1, right panel, we show a close up of the small-scale surface structure.

\section{Modeling Approach}

In this work, we use a simulation code based on RODS interactions. The method is thoroughly described by Muinonen et al. (2009), so here we give only a brief description. Diffraction and geometric optics are treated separately. Moreover, the RODS code takes into account internal and/or external diffuse scattering media. The diffuse scatterers can 

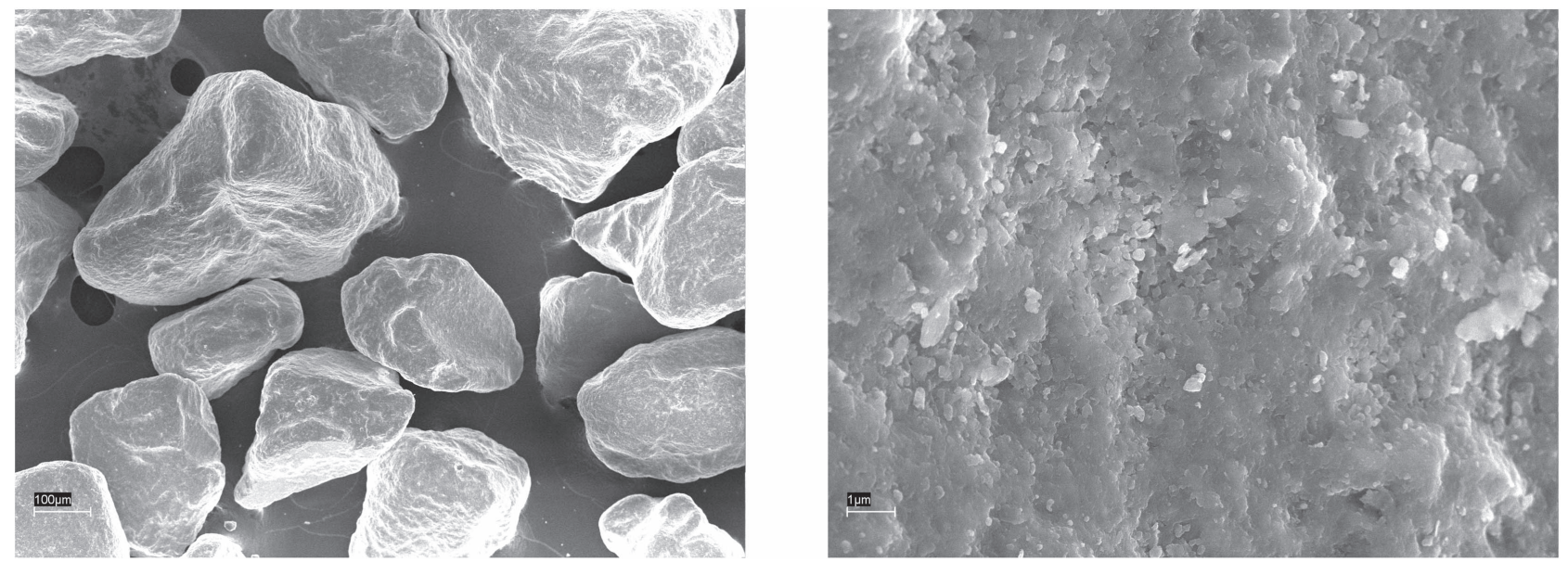

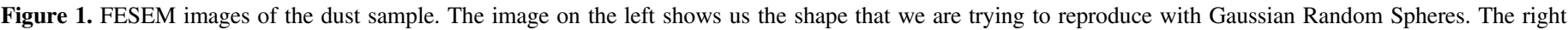
image shows wavelength-size surface roughness. The bar in the bottom left corner of each panel corresponds to $100 \mu \mathrm{m}$ and $1 \mu \mathrm{m}$ respectively.

Table 1

Physical Properties of Hematite and White Clay Samples

\begin{tabular}{|c|c|c|c|c|}
\hline & $m=n+i k$ & $r_{\text {eff }}$ & $v_{\text {eff }}$ & Composition \\
\hline Hematite & $3+i 0.01$ & 0.4 & 0.6 & $\mathrm{Fe}_{2} \mathrm{O}_{3}$ \\
\hline White Clay & $1.6+i 10^{-5}$ & 2.6 & 0.7 & Illite, kaolinite, montmorillonite, quartz \\
\hline
\end{tabular}

Note. These data are available at the Amsterdam-Granada light scattering database (www.iaa.es/scattering).

constitute an internal medium distributed uniformly within the particle interior (DIM). Moreover, they can be added as an external medium covering the surface of the particle (DEM). Whereas DIM is a three-dimensional medium, the physical thickness of DEM is negligible compared to the size of the particle, and diffuse scattering occurs within a single infinitesimal location on the surface. DIM is defined by the volume fraction $\rho_{\text {int }}$ and single-scattering albedo $\varpi_{\text {int }}$, while DEM is described by its optical thickness $\tau_{\text {ext }}$ and $\varpi_{\text {ext }}$. These inhomogeneities produce their own scattering pattern inside the host particle. The scattering behavior of these inclusions can be simulated inside RODS using either double Henyey-Greenstein functions, Rayleigh scattering, or customized input matrices. The latter case is the one selected in this work; the input matrices comprise six elements $\left(F_{11}(\theta), F_{12}(\theta)\right.$, $F_{22}(\theta), F_{33}(\theta), F_{34}(\theta)$, and $\left.F_{44}(\theta)\right)$ taken from laboratory measurements; these are the non-zero independent elements when the sample is an ensemble of irregular particles with random orientations.

The simulations discussed in this work were performed at $\lambda=632.8 \mathrm{~nm}$ so as to agree with the laboratory setup used to measure the input matrices, as well as the results of Nousiainen et al. (2011). Strictly speaking, the ROA is valid when the curvature of the particle surface is much larger than the wavelength of the incident radiation everywhere on the particle and the surface can thus be considered locally planar, and when the phase differences between internal and external fields across the surface irregularities are sufficiently large to suppress the interference effects associated with the irregularities (Muinonen et al. 1997). However, it may provide sufficiently accurate results even when these conditions are not fully met. It is noted that the lower particle size limit of the the ROA is not well defined and difficult to establish as it depends, for example, on particle shape and composition. As presented in the previous section, the dust sample consists of large particles with overall curvature radii much larger than the wavelength but the particles are partly covered with wavelength-scale surface roughness. That makes it an interesting test case for studying the performance of RODS.

\subsection{Input Matrices}

In this section we describe the samples used to mimic internal/external inhomogeneities. All optical properties given below are valid for a wavelength of $632.8 \mathrm{~nm}$. The scatterers are embedded in the host particle, which has refractive index $m_{\text {host }}=1.5+i(0.00001-0.0099)$. The imaginary part of the refractive index, $k$, is varied broadly by several orders of magnitude to test its role on the computed scattering matrix.

Based on the desertic origin of our cosmic dust analogue, we can assume hematite (Jeong \& Nousiainen 2014; Kemppinen et al. 2015) and clay particles (Banin et al. 1988; Orenberg \& Handy 1992) as good candidates for simulating its internal and external inhomogeneities. Hematite is also found on Mars (Kula \& Baldwin 2012), and is of interest in the study of the history of water on the planet. Also, it may be a component of exoplanetary atmospheres if their temperature allow its presence in solid form (Grenfell et al. 2010). Clay flakes can be found on the surface of Mars (Roush \& Orenberg 1996). In this case, clay is found also in the presence of pyroxenes (Poulet et al. 2005). As a result, the sample of white clay that has previously been studied in the laboratory can be used to represent surface roughness in the samples where clay is present. Moreover, hematite and white clay are good examples of strongly and weakly absorbing particles, respectively.

In this work we have used experimentally measured hematite and white clay scattering matrices as inputs in the code for the DIM and DEM. The measured scattering matrix for hematite is presented in Muñoz et al. (2006) at $\lambda=632.8 \mathrm{~nm}$. The data for these two measured samples are hosted at the 
Amsterdam-Granada Light Scattering Database (Muñoz et al. 2012) and are freely available to the community. In Table 1 we show some physical properties of this sample. Hematite is a type of iron oxide, $\mathrm{Fe}_{2} \mathrm{O}_{3}$, having a dark red powder aspect. We assume a refractive index $m_{\text {hematite }}=3+i 0.01$. The measured scattering of the white clay sample at $647 \mathrm{~nm}$ is presented in Muñoz et al. (2011). The difference in wavelength between this measurement and the simulations is small enough to have insubstantial consequences. This material is a white powder whose main constituents are illite, kaolinite, montmorillonite, and quartz. In our calculations we assume a refractive index $m_{\text {white clay }}=1.6+i 10^{-5}$ at visible wavelengths.

As well as these two samples, it is also interesting to study the effects of water ice as inclusion material and as a wavelength-scale surface roughness for its role in many different astrophysical environments. In outer space we can find ice as a coating or major component of dust particles, for example in comets, asteroids, satellites, and planetary atmospheres. Its existence as a coating (partially or totally) in protoplanetary dust has been proposed in small particles (as small as $\sim 20 \mu \mathrm{m}$ ) in Grigorieva et al. (2007). Since there are no experimental scattering matrices for ice available, we have used a Mie model in this case. We assume a spherical particle with a radius $r=0.5 \mu \mathrm{m}$ and refractive index $m_{\text {water ice }}=1.33+$ $i 10^{-5}$. From this Mie simulation, other optical parameters are obtained in addition to the scattering matrix, among them, the extinction coefficient $Q_{\text {ext }}$, which is necessary for computing the mean free path $l_{o}$ for the DIM inclusions (Equation (6)). These values are $Q_{\mathrm{ext}}=1.013$ when the host particle is weakly absorbing, and $Q_{\text {ext }}=0.471$ for the highly absorbing host particle. This $Q_{\text {ext }}$ has been computed using the relative refractive index $\left(m_{\text {water ice }} / m_{\text {host }}\right)$ as the host medium is not a vacuum. When the host particle is weakly absorbing, $k_{\text {host }}$ is small enough to neglect, so we only take into account $n_{\text {host }}$ (see Table 1). Since the classical Mie theory cannot be used for highly absorbing host media, we have used a code developed by Sudiarta $\&$ Chylek (2001) to obtain $Q_{\text {ext }}$ when $m_{\text {host }}=1.5$ $+i 0.0099$.

As in the case for ice, we need the value of $Q_{\text {ext }}$ to compute $l_{o}$ for hematite and white clay as an input for the DIM simulations. As the hematite particles are small enough, we assume the size distribution of the hematite measured in the laboratory by Muñoz et al. (2006) when computing the Mie simulation. We obtain a value of $Q_{\text {ext }}$ equal to 2.091 for $m_{\text {host }}=1.5+i 10^{-5}$ and 2.69 for $m_{\text {host }}=1.5+i 0.0099$. The same procedure is followed to compute $Q_{\text {ext }}$ for white clay, but we use $r=0.5 \mu \mathrm{m}$ inclusion radius as the measured white clay size distribution is too big to act as an internal inhomogeneity. The resulting value for $Q_{\text {ext }}$ is equal to 0.477 for $m_{\text {host }}=1.5+$ $i 10^{-5}$ and 0.375 for $m_{\text {host }}=1.5+i 0.0099$.

\subsection{Particle Shapes}

The host particle shapes were generated as GRSs, as detailed in Muinonen et al. (2009). In spherical coordinates, they are described by a radius vector which is the exponential of a Gaussian random variable:

$$
\begin{aligned}
& r(\vartheta, \phi) e_{r}=\frac{a \exp [s(\vartheta, \phi)]}{\sqrt{1+\sigma^{2}}} e_{r}, \\
& s(\vartheta, \phi)=\sum_{l=0}^{\infty} \sum_{m=-l}^{l} s_{\operatorname{lm}} Y_{\operatorname{lm}}(\vartheta, \phi),
\end{aligned}
$$

$$
s_{l,-m}=(-1)^{m} s_{\mathrm{lm}}^{*}
$$

where $s(\vartheta, \phi)$ is the logarithmic radial distance, $Y_{1 \mathrm{~m}}$ are orthonormal spherical harmonics, and $s_{1 \mathrm{~m}}$ are Gaussian random variables with zero means. The parameters $a$ and $\sigma$ are the mean and relative standard deviation. The standard deviation of the Gaussian random variables $s_{1 \mathrm{~m}}$ follows the covariance function $\Sigma_{s}$, which is given by a series of Legendre polynomials $P_{l}$. The degree $l$ of these polynomials ranges from 0 to $\infty$, but in the code the series is truncated by $l_{\min }$ and $l_{\max }$. The greater the value of $l_{\min }$ and $l_{\max }$, the spikier the particle will be, reducing its sphericity accordingly.

The code parameters for the GRS are the mean radius of the host particle $r, \sigma$ which describes the relative standard deviation of the radius vector, $\nu$ which sets the power law of the covariance function, and $l_{\min }$ and $l_{\max }$ to fix the coefficients of the Legendre polynomials and the correlation angle for autocorrelation. The values of these parameters (except the radius $r$ ) are fixed for all the simulations presented in this work: $\sigma=0.2, \nu=3.3, l_{\min }=2$, and $l_{\max }=11$. These values are in agreement with the shape distribution of the dust sample presented in Muñoz et al. (2007), which is used as the host particle in this work.

\section{Results}

As explained above, we analyze two different kinds of inhomogeneities through the addition of DIM or DEM to the host particle. These media can be composed of one of three materials: hematite, white clay, and water ice. For the DIM, the extent of its inclusion is determined by the volume fraction, $\rho_{\text {int }}$. For the case of the DEM, the equivalent parameter is the optical depth, $\tau_{\text {ext }}$. In both sets of simulations we have fixed the single-scattering albedos of the inclusions, $\varpi_{\text {int }}$, and surface roughness elements, $\varpi_{\text {ext }}$, to 0.9 .

Previous results (Muñoz et al. 2007) seem to indicate that the spikiness of the host particle, defined by $l_{\min }$, can actually mimic surface roughness but with less realistic shapes. Therefore, we decide to fix $l_{\min }=2$ corresponding to the actual shape of the dust particles and control surface roughness exclusively by varying $\tau_{\text {ext }}$.

Moreover, we have tested the sensitivity of the computed scattering matrices to the value of the real part of the refractive index $(n)$. The results (not shown here) do not indicate any significant effect on the computed scattering matrix elements when changing the value of $n$ from 1.5 to 1.7 in steps of 0.02 . Thus, $n$ is fixed to 1.5 in all our simulations.

Some computed results presented in this section are integrated over a size distribution. In those cases, the size distribution consists of 26 different size bins, ranging between $9.55 \mu \mathrm{m}$ (size parameter $x \sim 95)$ and $707.95 \mu \mathrm{m}(x \sim 7030)$, corresponding to the measured size distribution for the dust sample. For every size bin, we use 5000 different shapes.

\subsection{Sensitivity Study for Model Parameters}

In this section we analyze the effects of changes in volume fraction, $\rho_{\text {int }}$, for DIM, and optical thickness, $\tau_{\text {ext }}$, for DEM. Size effect figures are also presented, showing how the sensitivity of the scattering matrix to the choice of optical parameters is dependent on the host particle's radius. 

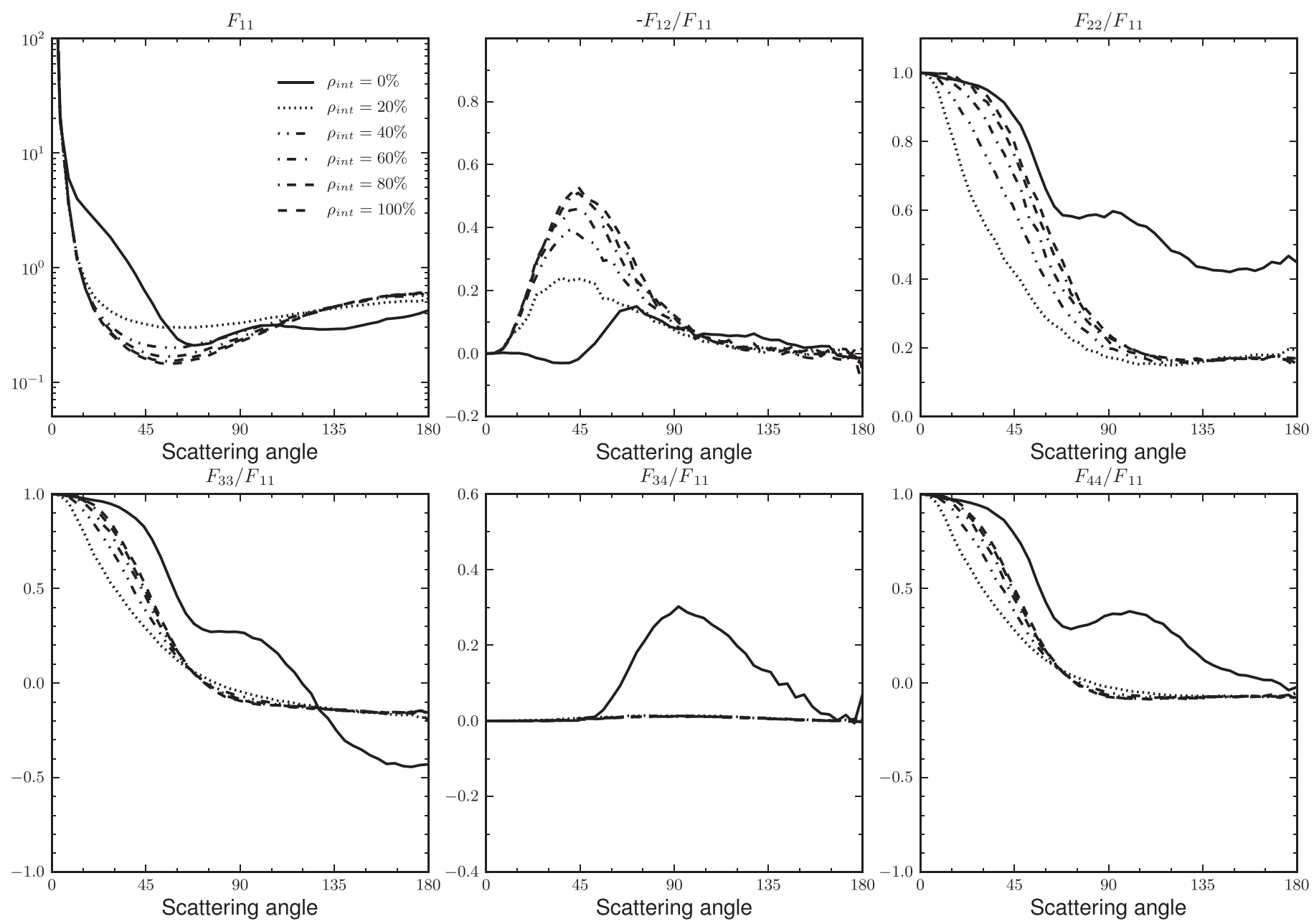

Figure 2. Effect of increasing the volume fraction, $\rho_{\text {int }}$, of hematite inclusions within a host particle of size parameter $x=100$ and with $m_{\text {host }}=1.5+i 10^{-5}$. Solid and dashed lines correspond to the two extreme cases, i.e., host particle without any inclusions $\left(\rho_{\text {int }}=0 \%\right)$ and a pure hematite host particle $\left(\rho_{\text {int }}=100 \%\right)$.

\subsubsection{Internal Inclusions}

In a first step we study the effect of switching on the DIM within RODS by changing the volume fraction, $\rho_{\text {int }}$, of the hematite (highly absorbing), white clay, and water ice (both weakly absorbing) inclusions in a weakly absorbing $\left(m_{\text {host }}=1.5+i 10^{-5}\right)$ host particle of constant size parameter $x=100$. We have studied the whole range of $\rho_{\text {int }}$ from $0 \%$ to $100 \%$. The scattering matrix elements computed for the hematite case $\left(m_{\text {inclusion }}=3+i 10^{-2}\right)$ are shown in Figure 2 . There are common trends with increasing $\rho_{\text {int }}$ across all three inclusion types, but these were most pronounced in the case of hematite.

In the case of the $F_{11}(\theta)$ element, it is interesting to note that increasing the volume fraction of internal inclusions produces a narrowing effect in the forward-scattering peak. Moreover, it produces a significant enhancement of the flux in the backwards hemisphere for all volume fractions studied. A higher volume fraction of inclusions also raises the peak of the degree of linear polarization for unpolarized incident light $\left(-F_{12}(\theta) / F_{11}(\theta)\right.$ ratio) and shifts it toward lower scattering angles. Again, this shifting effect is most pronounced in the case of the highly absorbing hematite inclusions, and is barely noticeable (although still present) for white clay and water ice inclusions. The $F_{33}(\theta) / F_{11}(\theta), \quad F_{34}(\theta) / F_{11}(\theta)$, and $F_{44}(\theta) / F_{11}(\theta)$ ratios tend to decrease at nearly all scattering angles when increasing $\rho_{\text {int }}$ for all three inclusion types. It is important to highlight that the $F_{34}(\theta) / F_{11}(\theta)$ ratio drops to 0 at all scattering angles.

In a second step, we assume a highly absorbing $\left(m_{\text {host }}=1.5\right.$ $+i 9.9 \times 10^{-3}$ ) host particle. Figure 3 shows the computed scattering matrix elements for different values of the volume fraction $(20 \%, 40 \%, 60 \%$, and $80 \%)$ for three highly absorbing host particles with different size parameters, namely $x=100$, 1000 , and 7000. In the above figure, the inclusions have the same refractive index as white clay $\left(m_{\text {inclusion }}=1.6+i 10^{-5}\right)$.

In contrast with the weakly absorbing host particle with high absorbing inclusions, the increase of $F_{11}(\theta)$ at the side- and back-scattering regions is significantly weaker. Nevertheless, we still see the effect when increasing the volume fraction of inclusions. Moreover, the increase in the maximum of the degree of linear polarization when increasing $\rho_{\text {int }}$ is not as strong as in the case of the weakly absorbing host particle. This increase of $\rho_{\text {int }}$ has hardly any effect for medium and large particles $(x=1000$ and 7000 respectively), and little for the small ones. The $F_{33}(\theta) / F_{11}(\theta)$ and $F_{44}(\theta) / F_{11}(\theta)$ ratios tend to increase when increasing the value of $\rho_{\text {int }}$ for all three inclusion types at the side- and back-scattering regions. The $F_{22}(\theta) / F_{11}(\theta)$ ratio decreases nearly at all scattering angles when increasing the value of $\rho_{\text {int }}$. As in the previous case, the $F_{34}(\theta) / F_{11}(\theta)$ ratio drops to 0 . 

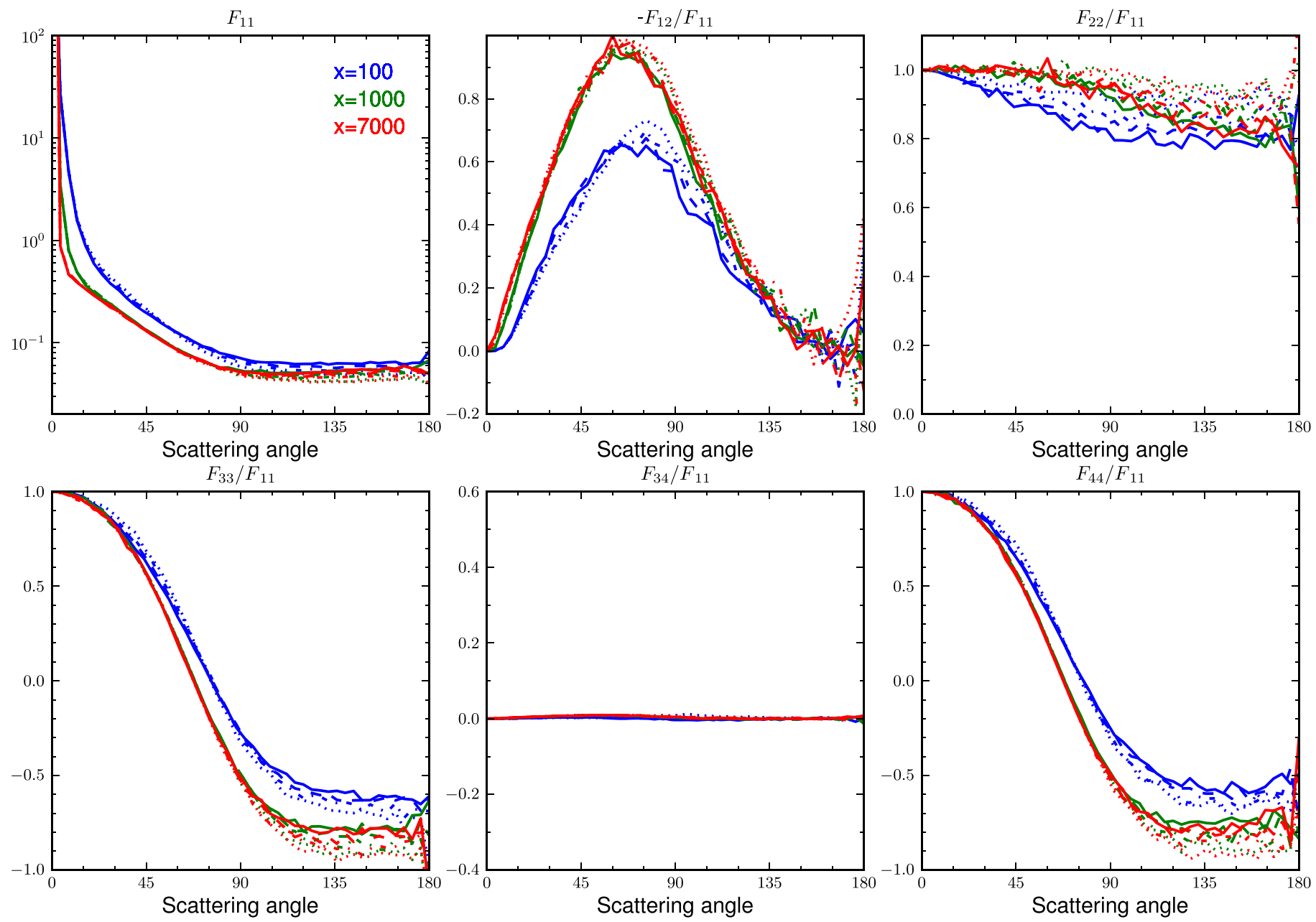

Figure 3. Effect of increasing the volume fraction, $\rho_{\text {int }}$, of white clay inclusions within a highly absorbing $\left(m_{\text {host }}=1.5+i 9.9 \times 10^{-3}\right.$ ) host particle, varying the size parameter $x=100,1000$, and 7000 (blue, green, and red lines, respectively). The volume fraction of internal inclusions, $\rho_{\text {int }}$, are $20 \%$ (dotted line), $40 \%$ (dotteddashed line), $60 \%$ (dashed line), and $80 \%$ (solid line).

In Figure 4 we present an equivalent study for a weakly absorbing host particle $\left(m_{\text {host }}=1.5+i 10^{-5}\right)$ with weakly absorbing white clay inclusions $\left(m_{\text {inclusion }}=1.6+i 10^{-5}\right)$. We also perform some simulations for another weakly absorbing host particle of $m_{\text {host }}=1.5+i 4 \times 10^{-5}$ to test the sensitivity to small changes of $k_{\text {host }}$, but we observe no differences. The effect of increasing $\rho_{\text {int }}$ is more remarkable for small sizes than for medium and large ones. As we can see, for size parameters $x=100$ and $x=1000$, the scattering matrix shows variability when increasing $\rho_{\text {int }}$, with the effect rapidly saturating when $x=7000$. The maximum of the $-F_{12}(\theta) / F_{11}(\theta)$ ratio is remarkably smaller for small particles compared with $x=1000$ and $x=7000$, and the $F_{22}(\theta) / F_{11}(\theta)$ ratio for these small particles tends to decrease when increasing $\rho_{\text {int }}$, the opposite behavior to that shown in Figure 3. The $F_{33}(\theta) / F_{11}(\theta)$ and $F_{44}(\theta) / F_{11}(\theta)$ ratios have a softer fall for small host particles.

In a third step, we study the effect of adding inclusions on the computed scattering matrix elements when integrating over a size distribution. Figure 5 shows the computed results for a size distribution of weakly absorbing $\left(m_{\text {host }}=1.5+\right.$ $i 4 \times 10^{-5}$ ) host particles with three different percentages of white clay inclusions. All computed scattering matrices with internal inclusions are presented together with the corresponding computed scattering matrix for a size distribution of "clean" host particles.

In general, the shape of all computed scattering matrix elements is smoothed out as we add inclusions. As in Figure 2, the forward-scattering peak width for the $F_{11}(\theta)$ element narrows, and a flattening effect appears at the side-scattering region. One of the most important effects of adding inclusions shows up in the maximum of the $-F_{12}(\theta) / F_{11}(\theta)$ ratio, which becomes significantly higher for hematite inclusions and moderately higher for white clay and ice inclusions. In general, the rest of the scattering elements have smaller values than the "clean" host particle case. The $F_{33}(\theta) / F_{11}(\theta)$ and $F_{44}(\theta) / F_{11}(\theta)$ ratios decrease when increasing $\rho_{\text {int }}$.

The effect of $\rho_{\text {int }}$ on the computed asymmetry parameter $g$ for a weakly absorbing host particle is shown in Figure 6. The trends observed are similar for all inclusion compositions. For hematite (left panel of the figure), increasing the volume fractions of inclusions tends to increase the asymmetry parameter for particles smaller than $147.91 \mu \mathrm{m}$ and does not produce any effect for larger particles. The same trend can be observed for white clay (middle panel), although the values of the computed asymmetry parameter are larger than for hematite, and the convergence occurs at $295.12 \mu \mathrm{m}$. The right panel shows a comparison between inclusion types with the same $\rho_{\text {int }}=30 \%$. Water ice and white clay inclusions have 

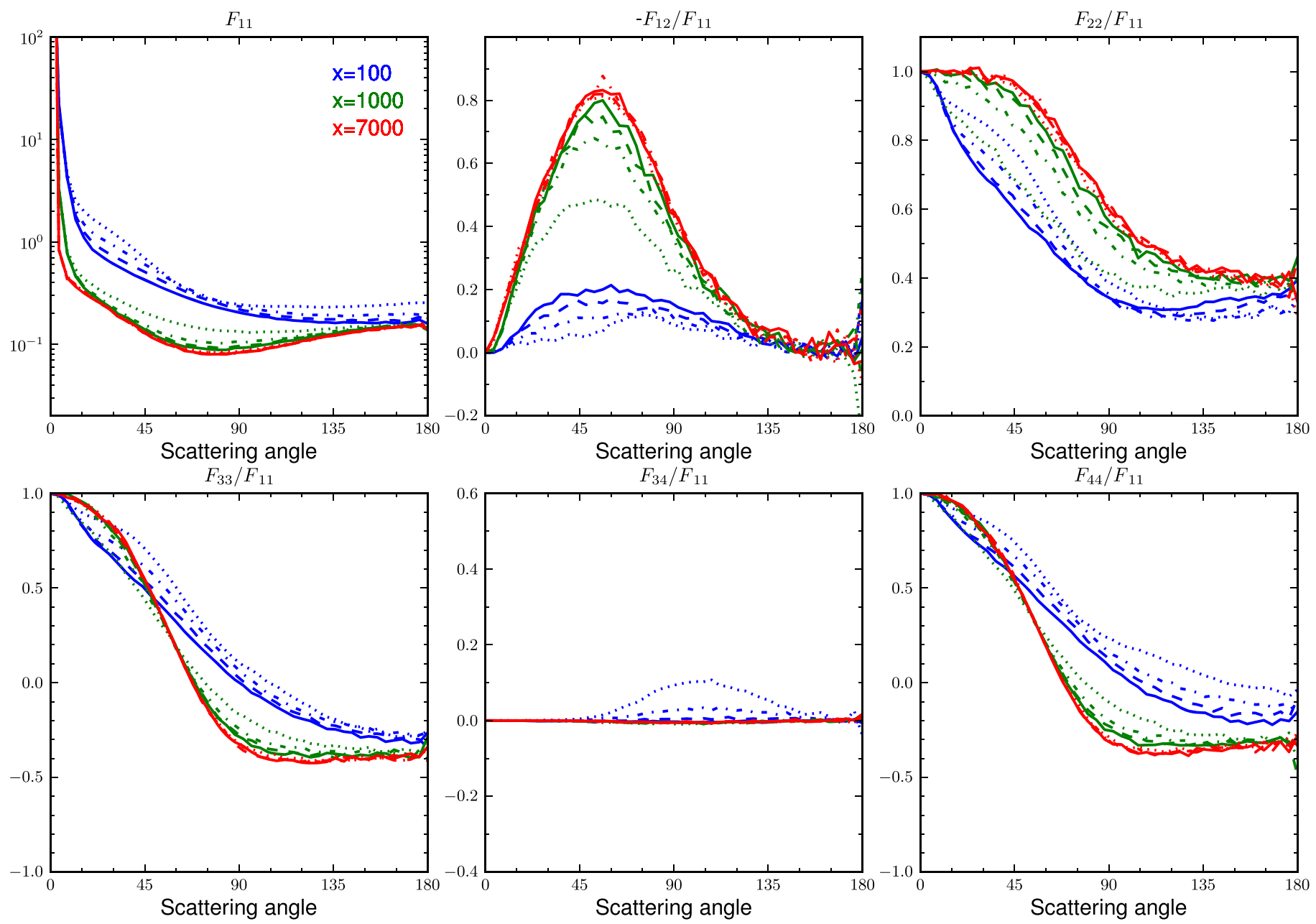

Figure 4. Effect of increasing the volume fraction, $\rho_{\text {int }}$, of white clay inclusions within a weakly absorbing $\left(m_{\text {host }}=1.5+i 10^{-5}\right)$ host particle, varying the size parameter $x=100,1000$, and 7000 (blue, green, and red lines, respectively). The volume fraction of internal inclusions, $\rho_{\text {int }}$, are $20 \%$ (dotted line), $40 \%$ (dotteddashed line), $60 \%$ (dashed line), and $80 \%$ (solid line).

virtually the same behavior, while hematite has smaller values for all sizes. Adding inclusions to the host particle size distribution triggers a flattening on the trend of the computed asymmetry parameter with scattering angle.

In Table 2 we present the computed single-scattering albedos $\varpi$ for a size distribution of a selected set of host particles, and the percentages and compositions of internal inclusions.

\subsubsection{Surface Roughness}

As presented in the previous section, in the first step we study the effect of switching on the DEM by changing the optical depth, $\tau_{\mathrm{ext}}$, of hematite (highly absorbing), white clay, and water ice (both weakly absorbing) surface roughness covering a weakly absorbing $\left(m_{\text {host }}=1.5+i 10^{-5}\right)$ host particle. The scattering matrix elements computed for the hematite case $\left(m_{\text {coating }}=3+i 10^{-2}\right)$ are shown in Figure 7.

As in Figure 2, the $F_{11}(\theta)$ element forward-scattering peak width narrows as $\tau_{\mathrm{ext}}$ increases, and the side-scattering and back-scattering regions flatten and increase over the clean host particle curve. A higher $\tau_{\text {ext }}$ decreases the maximum of the degree of linear polarization for unpolarized incident light $\left(-F_{12}(\theta) / F_{11}(\theta)\right.$ ratio). The $F_{22}(\theta) / F_{11}(\theta), \quad F_{33}(\theta) / F_{11}(\theta)$, $F_{34}(\theta) / F_{11}(\theta)$, and $F_{44}(\theta) / F_{11}(\theta)$ ratios tend to decrease at nearly all scattering angles when increasing $\tau_{\text {ext }}$ for all three surface roughness types, although this effect is stronger for the $F_{22}(\theta) / F_{11}(\theta)$ ratio.

In a second step, we assume a highly absorbing $\left(m_{\text {host }}=1.5\right.$ $+i 9.9 \times 10^{-3}$ ) host particle. Figure 8 shows the computed scattering matrix elements for various optical thicknesses $(0.2$, $0.4,0.6,0.8)$ for three highly absorbing host particles with different size parameters, namely $x=100,1000$, and 7000. For this figure, the surface roughness material has the same refractive index as white clay $\left(m_{\text {coating }}=1.6+i 10^{-5}\right)$.

As is known, the forward peak of the $F_{11}(\theta)$ element mainly depends on the size of the particles but not on the shape or composition (Liu et al. 2003). The larger the particles, the closer the forward peak is to $0^{\circ}$. It is interesting to note that, for a certain value $\tau_{\text {ext }}$, we do not find any significant effect on any of the elements of the scattering matrix when increasing the size parameter, with the exception of the $F_{11}(\theta)$ element in the forward direction. The computed degree of linear polarization $-F_{12}(\theta) / F_{11}(\theta)$ for a highly absorbing host particle seems to be very sensitive when increasing $\tau_{\text {ext }}$. The ratios $-F_{12}(\theta) / F_{11}(\theta)$ and $F_{22}(\theta) / F_{11}(\theta)$ tend to decrease when increasing $\tau_{\text {ext }}$, while the opposite effect occurs for the $F_{33}(\theta) / F_{11}(\theta)$ and $F_{44}(\theta) / F_{11}(\theta)$ ratios. The $F_{34}(\theta) / F_{11}(\theta)$ ratio is nearly insensitive to changes in $\tau_{\text {ext }}$ for all sizes.

For comparison we show the same results for a weakly absorbing host particle. As in the previous section, when 

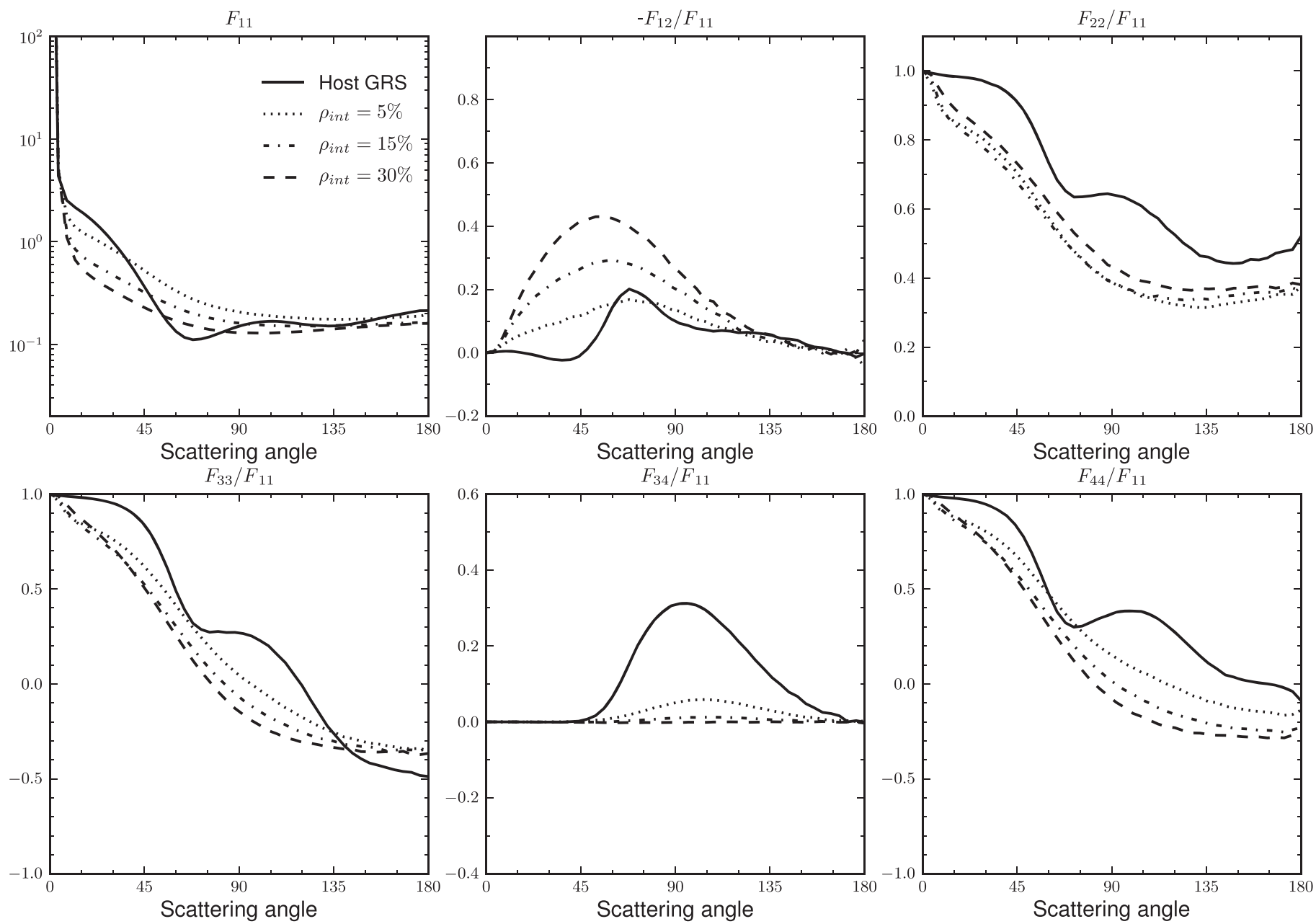

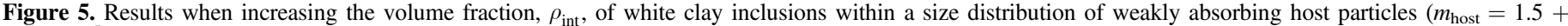

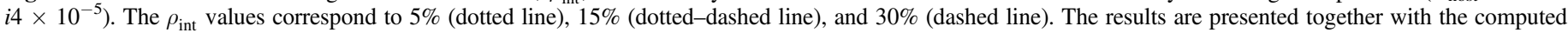
scattering matrix for a size distribution of clean host particles.
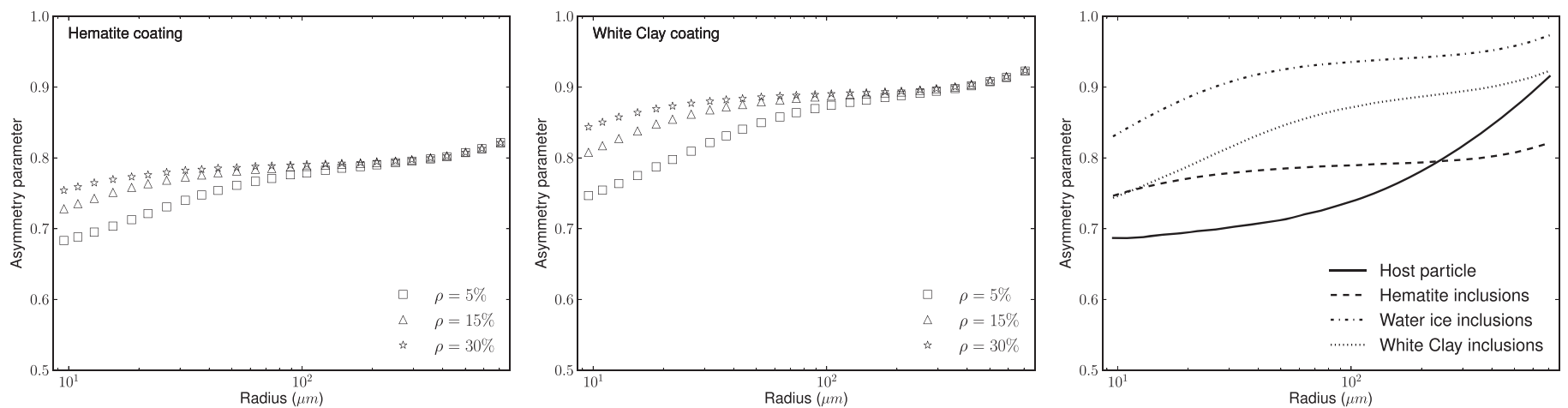

Figure 6. Effect on the computed asymmetry parameter when increasing the volume fraction of internal inclusions within a host particle with $m_{\text {host }}=1.5+i 10^{-5}$ : left panel for hematite inclusions and middle panel for white clay inclusions. The right panel shows a comparison between inclusion types (white clay, water ice, and hematite) with $\rho_{\text {int }}$ fixed to $30 \%$ and the "clean" host particles.

studying the internal inclusions, we perform two simulations of weakly absorbing host particles to test the sensitivity to small changes in the imaginary part of the refractive index (for $m_{\text {host }}=1.5+i 10^{-5}$ and $\left.m_{\text {host }}=1.5+i 4 \times 10^{-5}\right)$. This time we observe important changes. For $k_{\text {host }}=4 \times 10^{-5}$ (presented in Figure 9), the forward-scattering peak in $F_{11}(\theta)$ becomes more sensitive to changes in $\tau_{\mathrm{ext}}$ than the same simulations for $k_{\text {host }}=10^{-5}$. Moreover, in the case of $k_{\text {host }}=4 \times 10^{-5}$, we can see differences between sizes, where the largest size parameter $(x=7000)$ separates from the other two. Because of these reasons, and due to the fact that the dust sample has been characterized through a $k_{\text {host }}$ value of $4 \times 10^{-5}$, herein we discuss these results instead of those for $k_{\text {host }}=10^{-5}$. In contrast to the previous case shown in Figure 8, the scattering matrix elements are sensitive to the size of the host particle. The $F_{11}(\theta)$ forward-scattering region is more sensitive to changes in $\tau_{\text {ext }}$ for these weakly absorbing host particles than for the highly absorbing ones. The 
Table 2

Single-scattering Albedo $\varpi$ of the Simulated Size Distribution with DIM, for Three Different Types of Host Particles and Inclusion Composition as a Function of the Volume Fraction $\rho_{\text {int }}$

\begin{tabular}{|c|c|c|c|c|c|c|c|c|c|c|}
\hline \multirow{2}{*}{$m_{\text {host }}$} & \multicolumn{10}{|c|}{ Single-scattering Albedo $\varpi$ of a Size Distribution of Host Particles $m_{\text {host }}$ with Internal Inclusions } \\
\hline & $\rho_{\text {int }}=0$ & Hematite & White Clay & Water Ice & Hematite & White Clay & Water Ice & Hematite & White Clay & Water Ice \\
\hline $1.5+i 10^{-4}$ & 0.828 & 0.638 & 0.706 & 0.652 & 0.619 & 0.632 & 0.598 & 0.614 & 0.601 & 0.580 \\
\hline $1.5+i 4 \times 10^{-4}$ & 0.660 & 0.622 & 0.630 & 0.612 & 0.614 & 0.604 & 0.586 & 0.612 & 0.588 & 0.576 \\
\hline
\end{tabular}
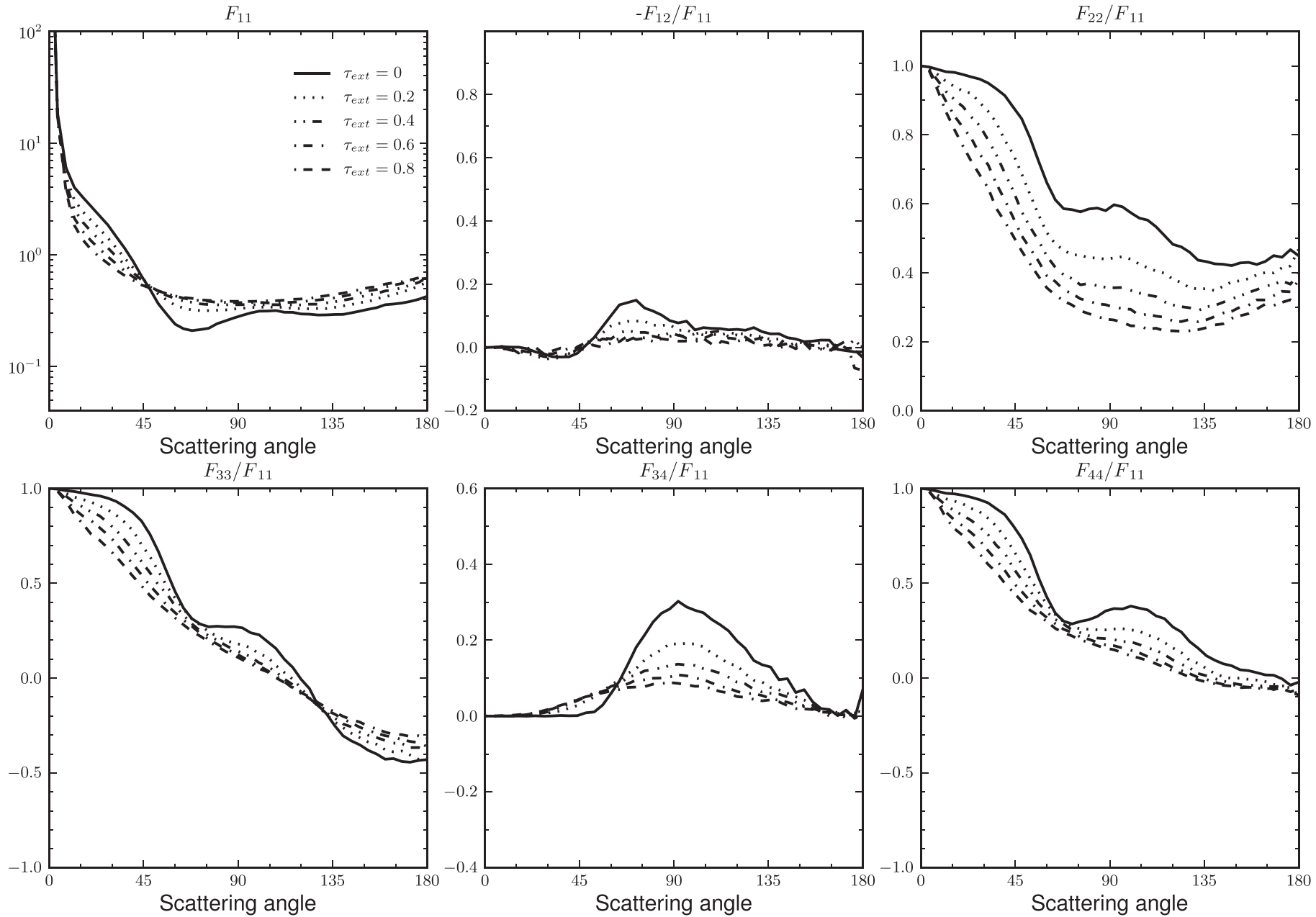

Figure 7. Effect of increasing the optical thickness, $\tau_{\text {ext }}$, of external scatterers composed of hematite over a host particle of size parameter $x=100$ and refractive index $m_{\text {host }}=1.5+i 10^{-5}$. Solid and dashed lines correspond to $\tau_{\mathrm{ext}}=0$ (clean host particle) and $\tau_{\mathrm{ext}}=0.8$.

$-F_{12}(\theta) / F_{11}(\theta)$ and $F_{22}(\theta) / F_{11}(\theta)$ ratios maintain the same trend as in Figure 8, but with softer changes. The $F_{33}(\theta) / F_{11}(\theta)$ and $F_{44}(\theta) / F_{11}(\theta)$ ratios are also less sensitive to changes in $\tau_{\text {ext }}$ than in a highly absorbing host particle. In contrast with Figure 8 , the $F_{34}(\theta) / F_{11}(\theta)$ ratio can trace variations in the surface roughness, decreasing its maximum when increasing $\tau_{\text {ext }}$.

In a third step, we study the effect of adding a coating on the computed scattering matrix elements when integrating over a size distribution. Figure 10 shows the computed results for a size distribution of weakly absorbing $\left(m_{\text {host }}=1.5+i 4 \times 10^{-5}\right)$ host particles with three different values of $\tau_{\text {ext }}$ for a white clay coating $\left(m_{\text {coating }}=1.6+\right.$ $i 10^{-5}$ ), although the same studies for hematite and water ice have been performed. All computed scattering matrices with surface roughness are presented together with the corresponding computed scattering matrix for a size distribution of "clean" host particles.

White clay and water ice have similar behaviors for $F_{11}(\theta)$. Again, the forward-scattering peak width narrows as $\tau_{\text {ext }}$ increases. Moreover, adding surface roughness flattens the phase function at side-scattering angles. The maximum of the $-F_{12}(\theta) / F_{11}(\theta)$ ratio (degree of linear polarization for unpolarized incident light) decreases in all cases when increasing the surface roughness. This effect is strongest for the hematite coating. The computed $F_{22}(\theta) / F_{11}(\theta)$, $F_{33}(\theta) / F_{11}(\theta)$, and $F_{44}(\theta) / F_{11}(\theta)$ ratios for hematite and white clay inclusions are similar to each other, their values decreasing 

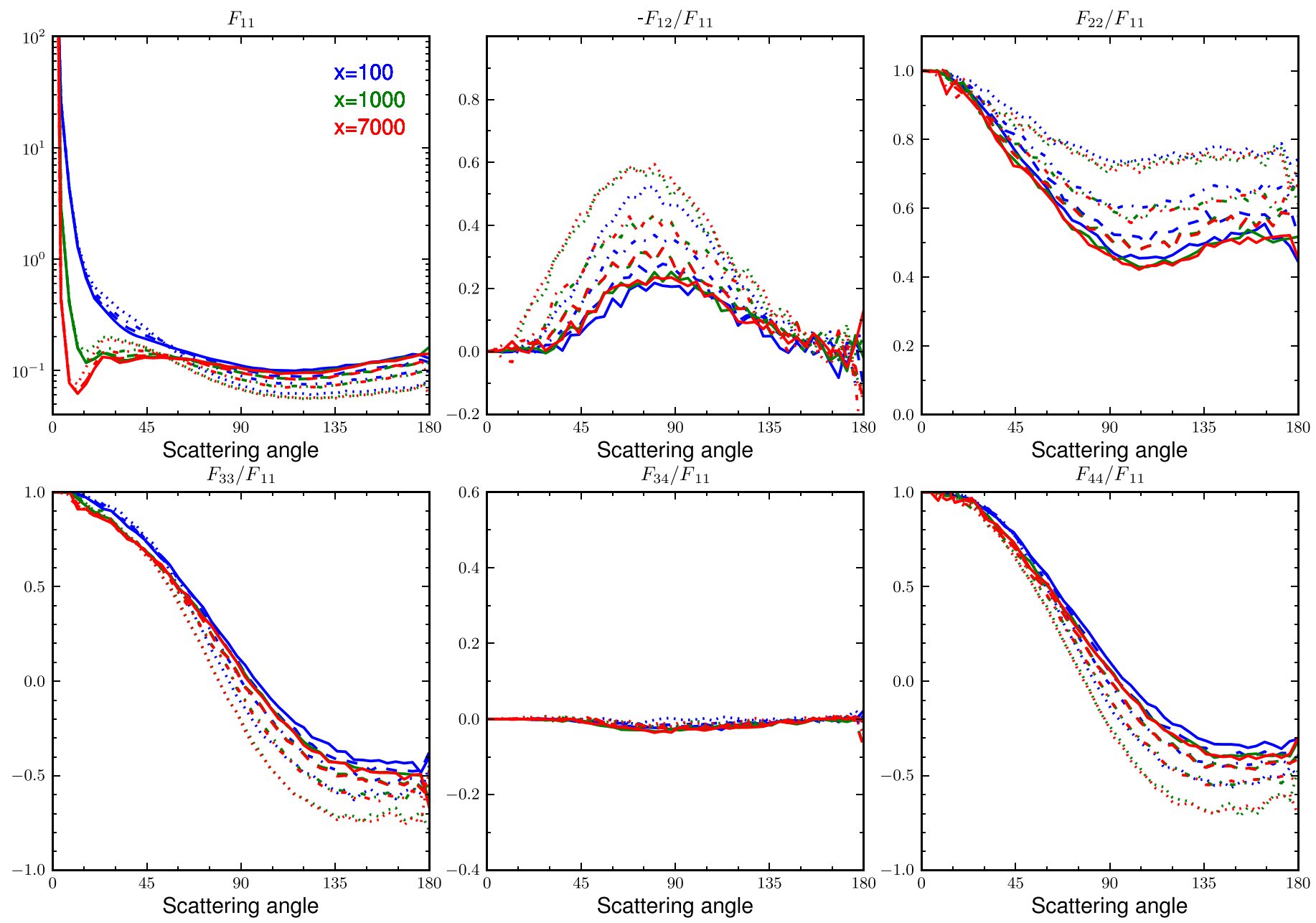

Figure 8. Effect of increasing the optical thickness, $\tau_{\text {ext }}$, of white clay acting as surface roughness over a highly absorbing $\left(m_{\text {host }}=1.5+i 9.9 \times 10^{-3}\right)$ host particle, varying the size parameter $x=100,1000$, and 7000 (blue, green, and red lines, respectively). Optical thickness $\tau_{\text {ext }}$ values are 0.2 (dotted line), 0.4 (dotted-dashed line), 0.6 (dashed line), and 0.8 (solid line).

with increasing $\tau_{\text {ext }}$. The $F_{22}(\theta) / F_{11}(\theta)$ ratio values for water ice are slightly closer to the clean host particle values than for the other two samples, for all $\tau_{\text {ext }}$. In the case of water ice surface roughness, the $F_{33}(\theta) / F_{11}(\theta)$ and $F_{44}(\theta) / F_{11}(\theta)$ ratios slightly increase with increasing $\tau_{\text {ext }}$, instead of the soft decrease shown in Figure 10.

Figure 11 shows the dependence of the asymmetry parameter, $g$, when varying the coating properties. As shown, $g$ increases as the particle size grows, due to the predominance of reflected light on the surface as the transmitted light is absorbed in the host particle, in agreement with previous results of Nousiainen et al. (2011). The left panel shows the results for a hematite coating, with $g$ decreasing for increasing $\tau_{\mathrm{ext}}$, while in the middle panel, for the white clay coating, there is almost no difference between different $\tau_{\text {ext }}$. In the right panel of Figure 11, we show that the computed results for a coating of white clay and water ice are very similar to each other and following the trend of the clean host particle size distribution, while the hematite coating has smaller values of the asymmetry parameter.

In Table 3 we present the computed single-scattering albedos $\varpi$ for a size distribution of a selected set of host particles, and the optical thicknesses and compositions for the surface roughness.

\subsection{Simulating the Experimental Scattering Matrices for Realistic Dust Particles}

As a final test for RODS, we consider the experimental scattering matrix for a realistic sample of dust particles presented in Muñoz et al. (2007). As mentioned above, we want to test the performance of RODS by including experimental scattering matrices for natural dust samples as internal and external scatterers.

In Figure 12 we present the measured scattering matrix elements as functions of the scattering angle for the dust sample. The measurements are performed at $632.8 \mathrm{~nm}$, covering the scattering angle range from $4^{\circ}$ to $174^{\circ}$. The measurements are presented together with (i) Mie computations for homogeneous spherical particles, (ii) RODS computations for "clean " GRS, and (iii) RODS computations for GRSs including a coating of white clay or hematite. In all computed cases we use the measured size distribution and refractive index $\left(m_{\text {host }}=1.5+i 4 \times 10^{-5}\right)$ of the dust sample. For comparison with the experimental data, all calculated phase functions are normalized to 1 at $30^{\circ}$. From the comparison of the experimental scattering matrix with Mie calculations, it is clear that light scattered by an ensemble of randomly oriented dust grains can be dramatically different from that scattered by an ensemble of spheres with the same refractive index and 

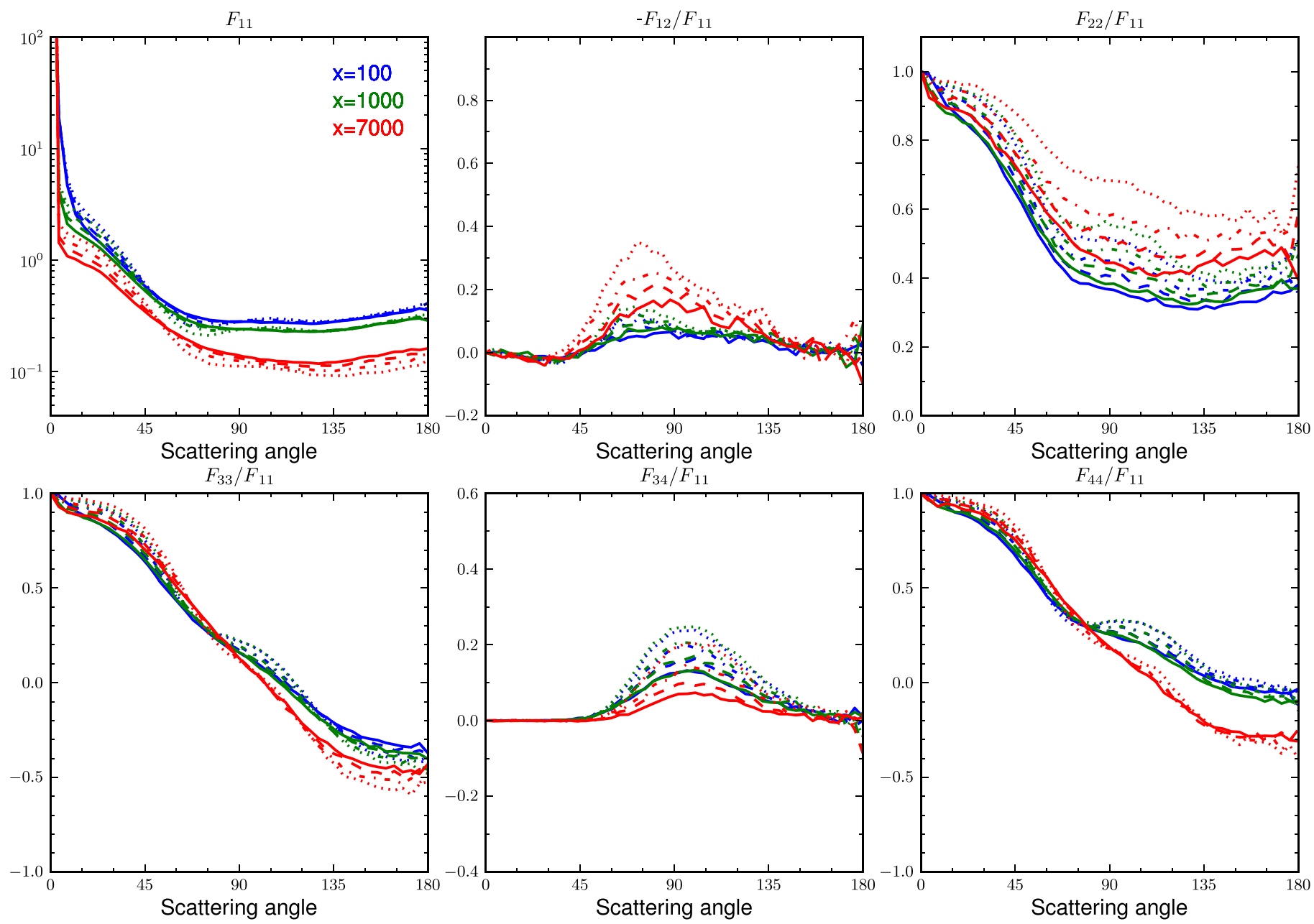

Figure 9. Effect of increasing the optical thickness, $\tau_{\text {ext }}$, of white clay acting as surface roughness over a weakly absorbing $\left(m_{\text {host }}=1.5+i 4 \times 10^{-5}\right)$ host particle, varying the size parameter $x=100,1000$, and 7000 (blue, green, and red lines, respectively). Optical thickness $\tau_{\text {ext }}$ values are 0.2 (dotted line), 0.4 (dotted-dashed line), 0.6 (dashed line), and 0.8 (solid line).

size distribution. The Mie identities $F_{11}(\theta)=F_{22}(\theta)$ and $F_{33}(\theta)=F_{44}(\theta)$ are not fulfilled by the dust sample particles. The measured phase function $F_{11}(\theta)$ for the dust sample presents a strong forward peak with a flat dependence at the side- and back-scattering regions. That seems to be a general characteristic of irregular compact dust grains. The degree of linear polarization for unpolarized incident light $\left(-F_{12}(\theta) / F_{11}(\theta)\right.$ ratio) for irregular dust grains shows a typical bell shape with a maximum around $90^{\circ}$ and a negative branch at large scattering angles. Moreover, the $F_{44}(\theta) / F_{11}(\theta)$ ratio tends to be larger than the $F_{33}(\theta) / F_{11}(\theta)$ ratio. The mentioned effects of non-sphericity may have serious implications when interpreting remote-sensing observations (e.g., Mishchenko et al. 2003; Min et al. 2012; Räisänen et al. 2013; Kahnert et al. 2014).

The effect of particle shape on the scattering matrix elements is clearly shown by the second set of simulations presented in Figure 12. All computed scattering matrix elements for a size distribution of "clean" GRSs are significantly closer to the experimental data than those computed for the same size distribution of spherical particles. However, the fitting may be further improved by taking internal and/or surface inhomogeneities of the dust grains into account. As examples, the third and the fourth sets of simulations in Figure 12 show the computed results for a size distribution of GRSs with a coating of white clay and hematite, respectively. In those simulations, we assume that the optical thickness, $\tau_{\mathrm{ext}}$, is equal to 1.0 and the single-scattering albedo, $\varpi_{\mathrm{ext}}$, is equal to 0.9 (Nousiainen et al. 2011). In general, adding surface roughness to the host particles improves the fit to the experimental data for roughly all scattering matrix elements. In particular, the coating of white clay produces nearly perfect fits to the experimental data for the $-F_{12}(\theta) / F_{11}(\theta), F_{22}(\theta) / F_{11}(\theta)$, and $F_{33}(\theta) / F_{44}(\theta)$ ratios. However, even though the shape of the $F_{11}(\theta)$ element is significantly improved in the case of the coating of white clay, we still do not get a perfect fit for that element.

Encouraged by the results of the sensitivity tests presented in the previous section, we try to improve the fitting to the experimental data including both DIM and DEM in our size distributions of GRS grains, corresponding to a more realistic model for natural dust. In Figure 13, we present the measured scattering matrix elements as functions of the scattering angle for the dust sample together with computations for a size distribution of "clean" GRSs and two different combinations of DIM and DEM, namely, $\mathrm{DIM}_{\text {hematite }}+\mathrm{DEM}_{\text {white clay }}$ and $\mathrm{DIM}_{\text {white clay }}+\mathrm{DEM}_{\text {white clay. }}$. In both cases, we fix $\rho_{\text {int }}=1 \%$ and $\varpi_{\text {int }}=0.9$, and $\tau_{\text {ext }}=0.5$ and $\varpi_{\mathrm{ext}}=0.9$, which are the parameters that best fit the measurements. As in the previous figure, in all computed cases we use the measured size distribution and refractive index $\left(m_{\text {host }}=1.5+i 4 \times 10^{-5}\right)$ of 

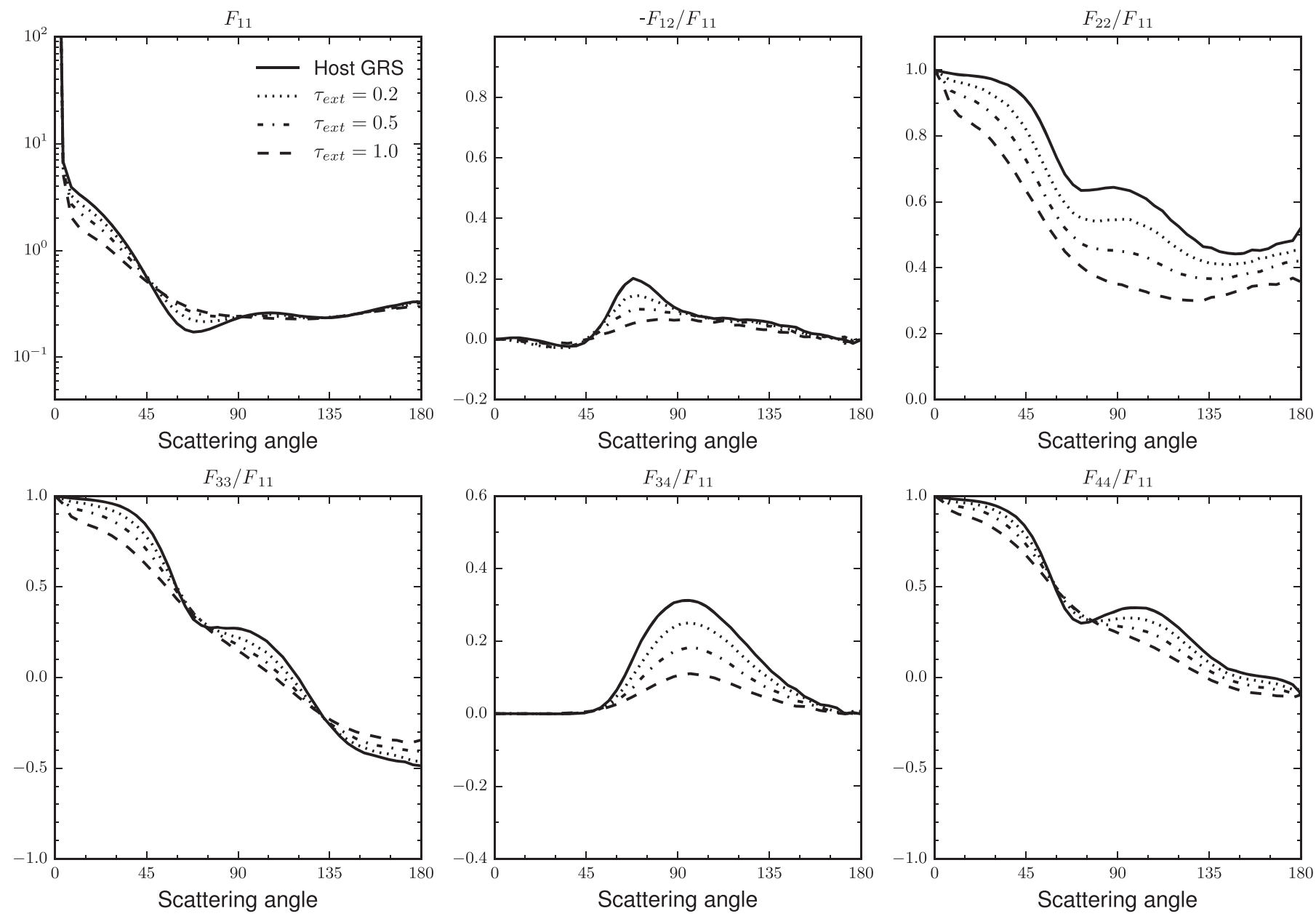

Figure 10. Results for increasing optical thickness, $\tau_{\text {ext }}$, of white clay as surface coating for a size distribution of weakly absorbing host particles $\left(m_{\text {host }}=1.5+\right.$ $i 4 \times 10^{-5}$ ). The $\tau_{\text {ext }}$ values correspond to 0.2 (dotted line), 0.5 (dotted-dashed line), and 1 (dashed line). The results are presented together with the computed scattering matrix for a size distribution of clean host particles.
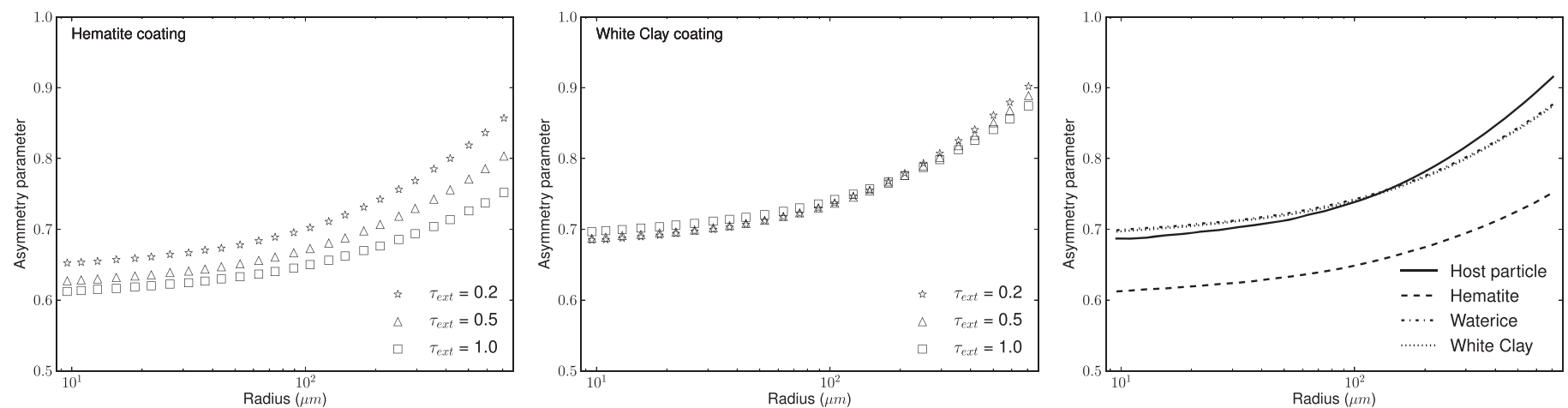

Figure 11. Computed asymmetry parameter as a function of radius for two coating materials, namely hematite (left panel) and white clay (middle panel), with different $\tau_{\text {ext }}$ values: 0.2 (stars), 0.5 (triangles), and 1.0 (squares). The bottom panel shows a comparison between coating samples: clean host particle (solid line), hematite (dashed line), water ice (chain-dashed line), and white clay (dotted line). In right panel $\tau_{\mathrm{ext}}=1$ and $\varpi_{\mathrm{ext}}=0.9$.

the dust sample. Moreover, all calculated phase functions are normalized to 1 at $30^{\circ}$. The combination of internal and external inhomogeneities produces better fits for almost all elements of the scattering matrix. With the combination $\mathrm{DIM}_{\text {white clay }}+\mathrm{DEM}_{\text {white clay }}$, we get a nearly perfect fit for the $-F_{12}(\theta) / F_{11}(\theta), F_{22}(\theta) / F_{11}(\theta)$, and $F_{33}(\theta) / F_{44}(\theta)$ ratios at all measured scattering angles. The $F_{11}(\theta) / F_{11}\left(30^{\circ}\right)$ ratio seems to be slightly overestimated at side- and back-scattering regions. However, we may note that this could be an effect of the arbitrary normalization to 1 at $30^{\circ}$. Figure 14 corresponds to the uppermost left panel of Figure 13, showing $F_{11}(\theta)$ with two types of normalizations: normalized to unity at $30^{\circ}$ and normalized to unity at $90^{\circ}$. This normalization at $90^{\circ}$ is used to avoid the forward-scattering domain where the $F_{11}(\theta)$ values show strong variations in a narrow range of scattering angles. As can be seen, the $\mathrm{DIM}_{\text {white clay }}+\mathrm{DEM}_{\text {white clay }}$ case produces a nearly perfect fit in the scattering angle range from $\sim 45^{\circ}$ to $174^{\circ}$. There is also good agreement in the diffraction peak 
Table 3

Single-scattering Albedo $\varpi$ of the Simulated Size Distribution with DEM, for Three Different Types of Host Particles and Surface Roughness Composition as a Function of the Optical Thickness $\tau_{\text {ext }}$

\begin{tabular}{|c|c|c|c|c|c|c|c|c|c|c|}
\hline \multirow{3}{*}{$m_{\text {host }}$} & \multicolumn{10}{|c|}{ Single-scattering Albedo $\varpi$ of a Size Distribution of Host Particles $m_{\text {host }}$ with Surface Roughness } \\
\hline & \multirow{2}{*}{$\tau_{\mathrm{ext}}=0$} & \multicolumn{3}{|c|}{$\tau_{\mathrm{ext}}=0.2$} & \multicolumn{3}{|c|}{$\tau_{\text {ext }}=0.5$} & \multicolumn{3}{|c|}{$\tau_{\text {ext }}=1$} \\
\hline & & Hematite & White Clay & Water Ice & Hematite & White Clay & Water Ice & Hematite & White Clay & Water Ice \\
\hline $1.5+i 10^{-4}$ & 0.828 & 0.808 & 0.804 & 0.804 & 0.783 & 0.774 & 0.804 & 0.749 & 0.734 & 0.734 \\
\hline $1.5+i 4 \times 10^{-4}$ & 0.660 & 0.665 & 0.653 & 0.653 & 0.669 & 0.645 & 0.644 & 0.673 & 0.633 & 0.632 \\
\hline
\end{tabular}
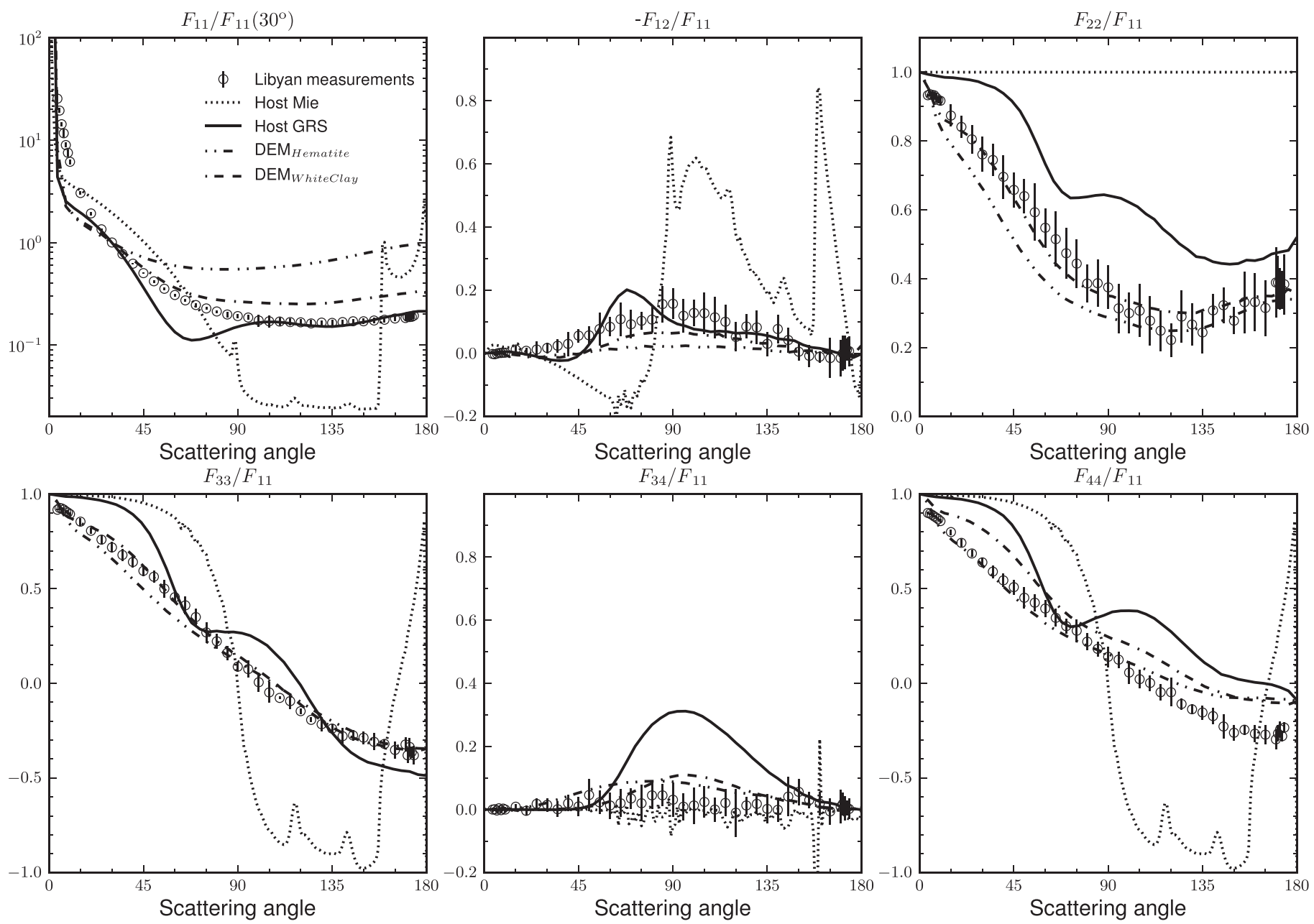

Figure 12. Comparison between experimental measurements of the dust sample size distribution (circles with error bars) and four different computed scattering matrices: a host particle size distribution composed of spheres computed by a Mie simulation (dotted line), a host particle size distribution of "clean" irregular particles (Gaussian Random Shapes, solid line), and two different size distributions of GRS particles with two surface roughness compositions: hematite (.--) and white clay $\left(-\right.$ (-). Surface roughness parameters are $\tau_{\mathrm{ext}}=1.0$ and $\varpi_{\mathrm{ext}}=0.9$.

regions, which is mainly dependent on the size of the particles. The main differences occur in the $5^{\circ}-45^{\circ}$ region where the computations underestimate the $F_{11}(\theta)$ element. The overall improvement to the fit with experimental data when inclusions are added illustrates the importance of the internal and surface structures when computing the scattering behavior of irregular particles.

\section{Summary and Conclusions}

The purpose of this work is to study the effects that internal inclusions and wavelength-scale surface roughness have on the scattering matrix elements of large cosmic dust grains. To achieve this, we perform sensitivity tests of the RODS code using experimentally measured scattering matrices as inputs for simulating internal and surface inhomogeneities. This paper can be considered as an extension of the study done by Nousiainen et al. (2011), but performing a parameter space exploration by changing the various knobs of the RODS code. For realistic input matrices, two different samples have been used: hematite and white clay, studied in Muñoz et al. (2006, 2010), respectively. These samples are chosen to compare the effect of highly absorbing and weakly absorbing inhomogeneities on the computed scattering matrix. Moreover, these materials are known to be present in astrophysical environments such as Mars, comets, and asteroids. 

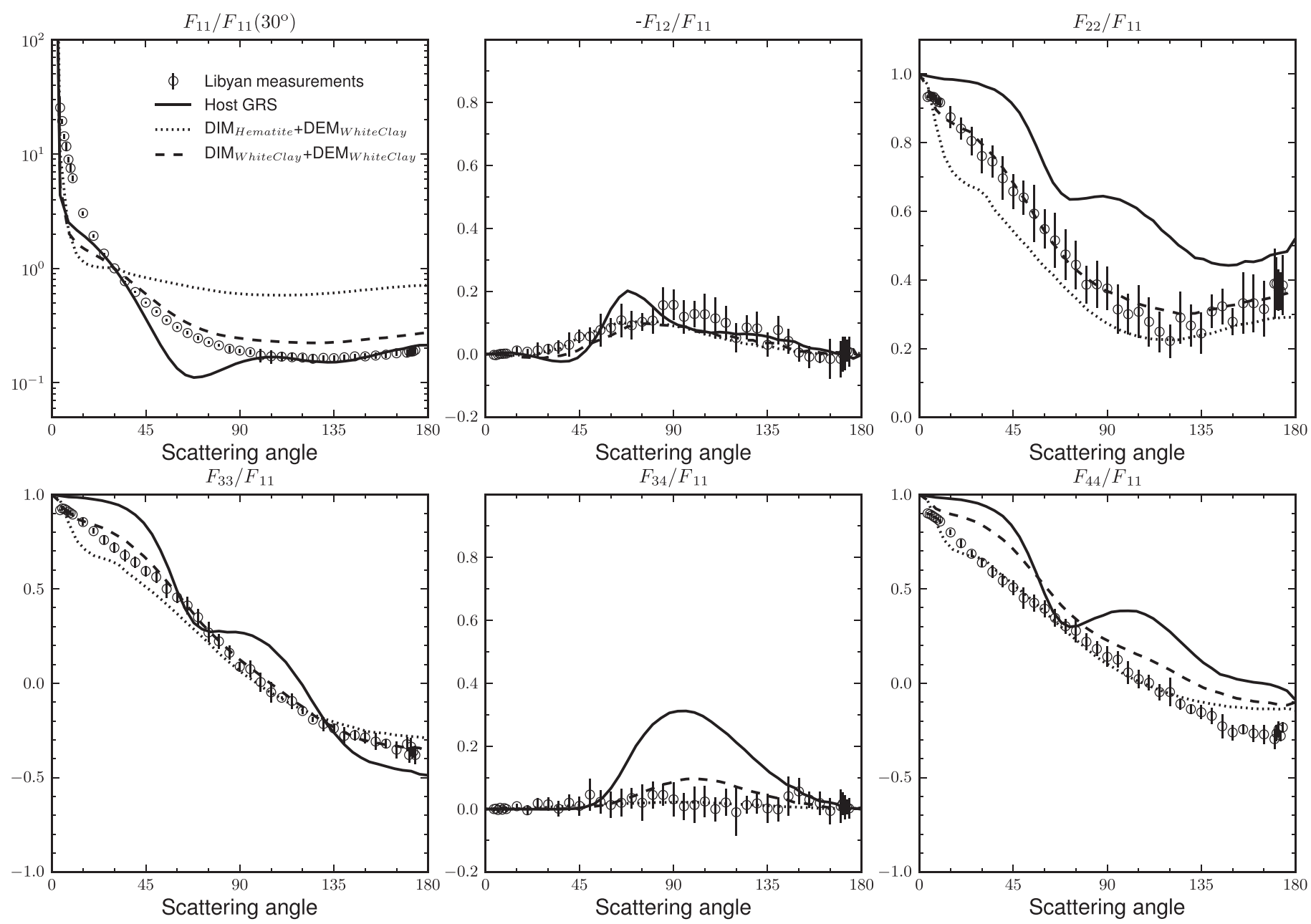

Figure 13. Comparison between experimental measurements of the dust sample size distribution (circles with error bars) and three different computed scattering matrices: a host particle size distribution of "clean" irregular particles (Gaussian Random Shapes, solid line) and two different size distributions of GRS particles including both Diffuse Internal Medium (DIM) and Diffuse External Medium (DEM) compositions, namely, DIM hematite + DEM $_{\text {white clay }}$ and $\mathrm{DIM}_{\text {white clay }}+\mathrm{DEM}_{\text {white clay. }}$. The DIM and DEM parameters are fixed, with $\rho_{\text {int }}=1 \%$ and $\varpi_{\text {int }}=0.9$, and $\tau_{\text {ext }}=0.5$ and $\varpi_{\text {ext }}=0.9$, respectively.

In particular we study the effects of changes in the volume fraction, $\rho_{\text {int }}$, of internal inclusions (DIM), and the optical thickness, $\tau_{\text {ext }}$, of the surface roughness (DEM). Size effect figures are also presented, showing how the sensitivity of the scattering matrix to the choice of optical parameters is dependent on the host particle's radius.

From the size effect studies, we conclude that the DIM hardly affects scattering when the host particle is highly absorbent. Sensitivity to DIM is practically non-existent regardless of the inclusion material. Only the $F_{11}(\theta)$, $-F_{12}(\theta) / F_{11}(\theta)$, and $F_{22}(\theta) / F_{11}(\theta)$ ratios are slightly affected for small host particle sizes. In contrast, when dealing with a weakly absorbing host particle, all scattering matrix elements are affected to some extent even when the DIM is composed of a weakly absorbing material. This situation is reversed when DEM is studied, as the computed scattering matrix elements for highly absorbing host particles are strongly affected when $\tau_{\text {ext }}$ increases. The effect of $\tau_{\mathrm{ext}}$ is mainly constrained to the $F_{11}(\theta)$, $-F_{12}(\theta) / F_{11}(\theta)$, and $F_{22}(\theta) / F_{11}(\theta)$ ratios. There are hardly any differences between different size parameters when studying DEM for $m_{\text {host }}=1.5+i 10^{-5}$ and $m_{\text {host }}=1.5+i 9.9 \times 10^{-3}$. Further simulations were performed for small differences in the imaginary part of the refractive index of the weakly absorbing host particle $\left(k_{\text {host }}=10^{-5}\right.$ and $\left.k_{\text {host }}=4 \times 10^{-5}\right)$. Even for such small differences in the value of $k_{\text {host }}$, we see a significant effect on the computed scattering matrix elements. In the case of $k_{\text {host }}=10^{-5}$, no size effect is observed for the studied size parameters $(x=100,1000$, and 7000). However, for $k_{\text {host }}=4 \times 10^{-5}$, the computed scattering matrix elements for the largest size parameter $(x=7000)$ show significant differences with the computed values for $x=100$ and 1000 .

From the results obtained for a size distribution of host particles, we consistently observe four effects as we add internal inclusions:

1. The forward-scattering peak width of the $F_{11}(\theta)$ element narrows as the volume fraction of inclusions increases.

2. In general, the F11 element is flattened in the sidescattering region and enhanced in the backwards hemisphere with increasing volume fractions of internal inclusions, being significantly stronger for a weakly absorbing host particle with highly absorbing inclusions (Figure 2); this is an interesting result for astronomy, as similar effects have been detected, e.g., in comet coma observations (e.g., Meech \& Jewitt 1987) and protoplanetary debris disks (e.g., Min et al. 2016).

3. The $F_{34}(\theta) / F_{11}(\theta)$ ratio rapidly decreases to 0 for growing $\rho_{\text {int }}$. 

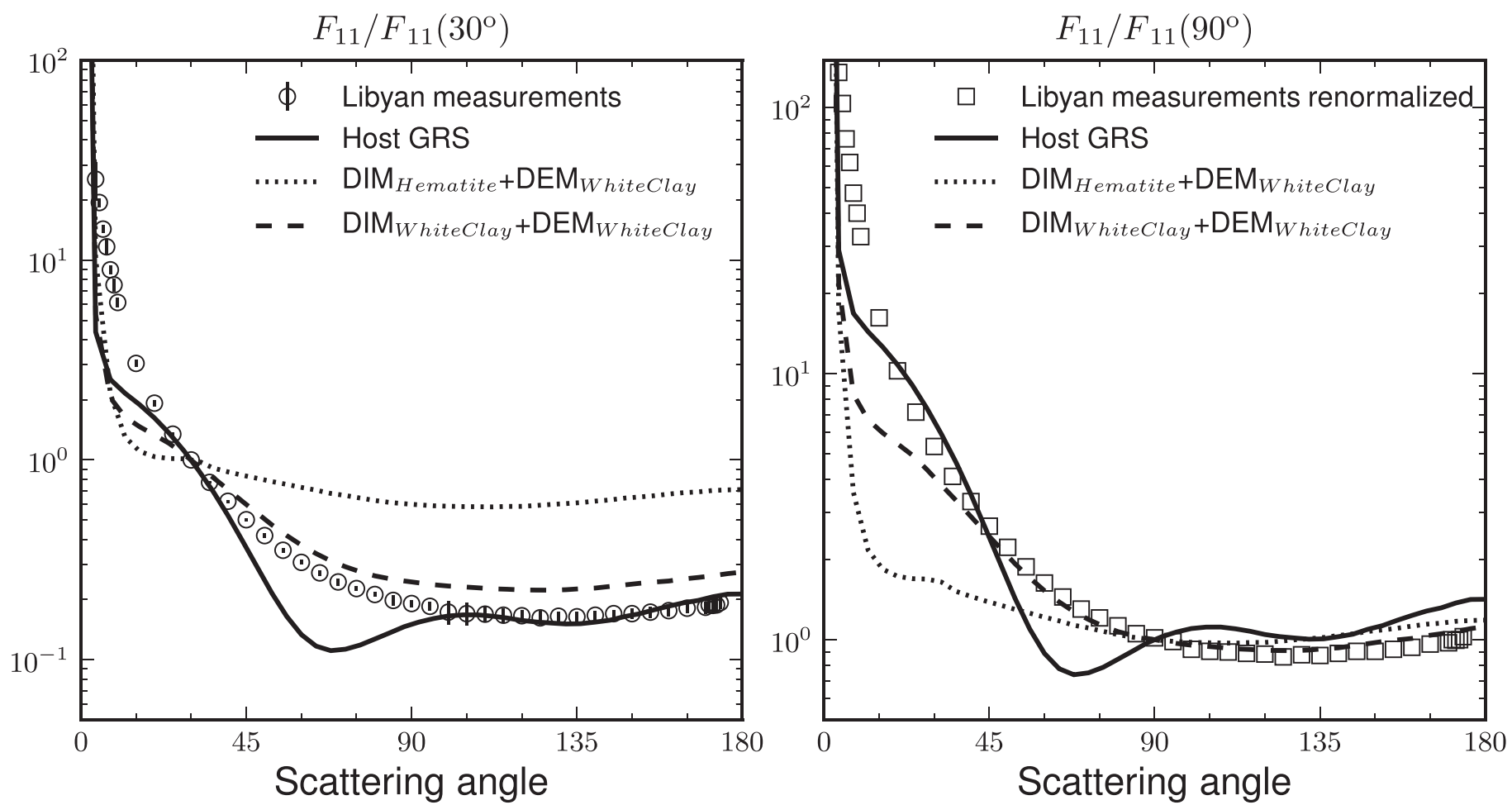

Figure 14. Comparison of the phase functions $F_{11}(\theta)$ with and without normalization. The left panel corresponds to the uppermost left panel of Figure 13 for the phase function $F_{11}(\theta) / F_{11}\left(30^{\circ}\right)$. The right panel displays the phase functions normalized at $90^{\circ}, F_{11}(\theta) / F_{11}\left(90^{\circ}\right)$. New normalized experimental data values are represented as empty squares in the right panel.

4. The maximum of the degree of linear polarization for unpolarized incident light $\left(-F_{12}(\theta) / F_{11}(\theta)\right.$ ratio $)$ increases strongly as the volume fraction of inclusions is increased (e.g., for the weakly absorbing host particle, the $-F_{12}(\theta) / F_{11}(\theta)$ ratio grows from $\approx 20 \%$ for the clean host particle to values over $40 \%$ when adding inclusions).

The $-F_{12}(\theta) / F_{11}(\theta)$ ratio increase could be due to a "darkening" within the host particle because of the addition of inclusions. In these tests, the host particles lack surface roughness, so the reflection of the incident light over the soft surface generates a strong linear polarization. Taking into account that for a host particle without internal inclusions, scattered light from the interior of the particle is mainly unpolarized, the degree of linear polarization for unpolarized incident light $\left(-F_{12}(\theta) / F_{11}(\theta)\right.$ ratio) reaches a maximum value of around 0.2. But when adding inclusions, the mean free path of the refracted light increases inside the particle, so the unpolarized refracted light that should compensate for the highly linearly polarized reflected light is extinguished, resulting in a higher degree of linear polarization. This effect can also be observed in the case of a highly absorbing host particle with weakly absorbing inclusions, because the majority of the inner volume is already absorbing.

The general features observed when adding surface roughness to a size distribution of host particles are:

1. The same narrowing of the $F_{11}(\theta)$ forward peak and flattening at the side- and back-scattering regions mentioned above.

2. A decrease in the degree of linear polarization for unpolarized light $\left(-F_{12}(\theta) / F_{11}(\theta)\right.$ ratio).

3. A smoothing and decrease of the rest of the scattering matrix elements.
Finally, we compare the measured scattering matrix for a dust sample presented in Muñoz et al. (2007) with Mie computations for homogeneous spherical particles, RODS computations for "clean" GRSs, and RODS computations for GRSs, including a coating of white clay or hematite. In all computed cases, we use the measured size distribution and refractive index $\left(m_{\text {host }}=1.5+i 4 \times 10^{-5}\right)$ of the dust sample. The Mie computations cannot reproduce any of the experimental scattering matrix elements. Computations for a size distribution of GRSs significantly improve the results. The fitting to the experimental data is further improved by taking internal and/or surface inhomogeneities of the dust grains into account. Indeed, our results seem to indicate that a small amount of internal inclusions $\left(\rho_{\text {int }}=1 \%\right)$ can significantly affect the computed scattering matrix elements.

In conclusion, using experimental scattering matrices to characterize surface roughness and internal inclusions provides an excellent approach to reproduce the scattering matrices of large cosmic dust grains. Both kinds of inhomogeneities play a major role in the scattering matrix elements. Some refinements are still needed in the code to better reproduce the experimental phase functions of cosmic dust grains at all scattering angles.

We gratefully acknowledge the comments on an earlier version of this paper by an anonymous referee. This work been supported by the Plan Nacional de Astronomía y Astrofísica contracts AYA2015-67152-R and AYA2015-71975-REDT.

\section{References}

Banin, A., Margulies, L., Ben-Shlomo, T., et al. 1988, Lunar Planet. Sci., 19,27

Della Corte, V., Rotundi, A., Fulle, M., et al. 2015, A\&A, 583, A13

Dorschner, J., Begemann, B., Henning, T., et al. 1995, A\&A, 300, 503 
Draine, D. T., \& Flatau, P. J. 1994, JOSAA, 11, 1491

Dubovik, O., Sinyuk, A., Lapyonok, T., et al. 2006, JGR, 111, D11

Fujiwara, H., Onaka, T., Ishihara, D., et al. 2010, ApJL, 714, L152

Fulle, M., Della Corte, V., Rotundi, A., et al. 2015, ApJL, 802, L12

Fulle, M., Marzari, F., Della Corte, V., Fornasier, S., et al. 2016, ApJ, 821, 1

Grenfell, J. L., Stock, J. W., Patzer, A. B. C., et al. 2010, P\&SS, 58, 1252

Grigorieva, A., Thébault, Ph., Artymowicz, P., et al. 2007, A\&A, 475, 755

Hansen, J. E., \& Travis, L. D. 1974, SSRv, 16, 527

Hovenier, J. W., van der Mee, C. V. M., \& Domke, H. 2004, Transfer of Polarized Light in Planetary Atmospheres: Basic Concepts and Practical Methods (Dordrecht, Berlin: Kluwer, Springer)

Jeong, G., \& Nousiainen, T. 2014, ACP, 14, 7233

Kahnert, F. M. 2003, JQSRT, 79, 775

Kahnert, M., Nousiainen, T., \& Lindqvist, H. 2014, JQSRT, 146, 41

Kemppinen, O., Nousiainen, T., \& Lindqvist, H. 2015, JQSRT, 150, 55

Kula, J., \& Baldwin, S. L. 2012, P\&SS, 67, 101

Liu, L., Mishchenko, M., Hovenier, J. W., et al. 2003, JQSRT, 79-80, 911

Meech, K. J., \& Jewitt, D. C. 1987, A\&A, 187, 585

Mie, G. 1908, AnPhy, 25, 377

Min, M., Canovas, H., Mulders, G. D., et al. 2012, A\&A, 537, A75

Min, M., Hovenier, J., \& de Koter, A. 2005a, A\&A, 432, 909

Min, M., Hovenier, J., de Koter, A., et al. 2005b, Icar, 179, 158

Min, M., Kama, M., Dominik, C., \& Waters, L. 2010, A\&A, 509, L6

Min, M., Rab, C., Woitke, P., et al. 2016, A\&A, 585, A13

Mishchenko, M., Geogdzhayev, I., Liu, L., et al. 2003, JQSRT, 79-80, 953

Mishchenko, M. I. 2009, JQSRT, 110, 808

Mishchenko, M. I., Hovenier, J. W., \& Travis, L. D. 2000, Light Scattering by Non spherical Particles: Theory, Measurements, and Applications (San Diego: Academic)
Molster, F. J., Waters, L. B. F. M., \& Tielens, A. G. G. M. 2002, A\&A, 382,222

Muinonen, K., Lamberg, L., Fast, P., et al. 1997, JQSRT, 57, 197

Muinonen, K., Nousiainen, T., Fast, P., et al. 1996, JQSRT, 55, 577

Muinonen, K., Nousiainen, T., Lindqvist, H., et al. 2009, JQSRT, 110 , 1628

Muñoz, O., Moreno, F., Guirado, D., et al. 2010, JQSRT, 111, 187

Muñoz, O., Moreno, F., Guirado, D., et al. 2011, Icar, 211, 894

Muñoz, O., Moreno, F., Guirado, D., et al. 2012, JQSRT, 113, 565

Muñoz, O., Volten, H., Hovenier, J. W., et al. 2006, A\&A, 446, 525

Muñoz, O., Volten, H., Hovenier, J. W., et al. 2007, JGR, 112, D13215

Nousiainen, T. 2009, JQSRT, 110, 1261

Nousiainen, T., Muinonen, K., \& Räisänen, P. 2003, JGR, 108, 4025

Nousiainen, T., Munoz, O., Lindqvist, H., et al. 2011, JQSRT, 112, 420

Orenberg, J., \& Handy, J. 1992, Icar, 96, 219

Patterson, E., Gillete, D., \& Stockton, B. 1977, JGR, 82, 3153

Poulet, F., Bibring, J., Mustard, J., et al. 2005, Natur, 438, 623

Räisänen, P., Haapanala, P., Chung, C. E., et al. 2013, QJRMS, 139, 2222

Roush, T. L., \& Orenberg, J. B. 1996, JGR, 101, 26111

Schröder, S., Grynko, Y., Pommerol, A., et al. 2014, Icar, 239, 201

Shettle, E., \& Fenn, R. 1979, AFCRL Tech. Rep., 79, 0214

Sudiarta, I. W., \& Chylek, P. 2001, JQSRT, 70, 709

Testi, Ł., Birnstiel, T., Ricci, L., et al. 2014, in Protostars and Planets VI, ed. H. Beuther et al. (Tucson, AZ: Univ. Arizona Press)

Wolff, M. J., Clancy, R. T., Goguen, J. D., et al. 2006, JGR, 111, 12

Wolff, M. J., Clancy, R. T., Goguen, J. D., et al. 2010, Icar, 208, 143

Zubko, E., Muinonen, K., Muñoz, O., et al. 2013, JQSRT, 131, 175 